\title{
Geometric quantum information structure in quantum fields and their lattice simulation
}

\author{
Natalie Klco $\odot^{*}$ and Martin J. Savage $\odot^{\dagger}$ \\ Institute for Nuclear Theory, University of Washington, Seattle, Washington 98195-1550, USA
}

(Received 24 September 2020; accepted 2 February 2021; published 22 March 2021)

\begin{abstract}
An upper limit to distillable entanglement between two disconnected regions of massless noninteracting scalar field theory has an exponential decay defined by a geometric decay constant. When regulated at short distances with a spatial lattice, this entanglement abruptly vanishes beyond a dimensionless separation, defining a negativity sphere. In two spatial dimensions, we determine this geometric decay constant between a pair of disks and the growth of the negativity sphere toward the continuum through a series of lattice calculations. Making the connection to quantum field theories in three-spatial dimensions, assuming such quantum information scales appear also in quantum chromodynamics (QCD), a new relative scale may be present in effective field theories describing the low-energy dynamics of nucleons and nuclei. We highlight potential impacts of the distillable entanglement structure on effective field theories, lattice QCD calculations and future quantum simulations.
\end{abstract}

DOI: 10.1103/PhysRevD.103.065007

\section{INTRODUCTION}

It is well known that the vacuum state of quantum fields exhibits entanglement between spatially separated regions [1-5]. Techniques for extracting this entanglement to auxiliary quantum systems through harvesting and subsequent distillation have been developed for a variety of relativistic fields, in some instances employing accelerating observers to causally disconnect the entanglement detectors [6-10]. This fundamental property of nature may prove useful in the distribution of entangled pairs through local interaction with a background field for quantum communications, sensing, or metrology as well as in providing new perspectives on the structure of spacetime [11-16].

Recent progress in quantum information has inspired increased consideration of entanglement in high-energy physics and nuclear physics processes. There have been a number of earlier studies examining the role of entanglement in dynamical processes related to high-energy quantum chromodynamics (QCD), such as fragmentation [17-19], heavy-ion collisions [20-23], deep inelastic scattering $[24,25]$ and even suggestive hints extracted from experimental data [26]. Further, some exciting results have recently been obtained connecting entanglement to emergent symmetries of QCD [27,28] and to the structure of nuclei [29-31].

\footnotetext{
*klcon@uw.edu;

Present address: Institute for Quantum Information and Matter and Walter Burke Institute for Theoretical Physics, California Institute of Technology, Pasadena, CA 91125, USA.

mjs5@uw.edu
}

In this work, we calculate the geometric constant determining the exponential component of the decay of negativity in the two-dimensional noninteracting massless scalar field vacuum. We further explore the structure of entanglement in the lattice-regulated field to inform the design of quantum and classical calculations of quantum coherent observables.

The choice of scalar field is inspired by its simplicity, ubiquity, unique status of having a thoroughly examined qubit digitization [32-36], and having been proven to be $\mathrm{BQP}$ (bounded-error quantum polynomial time) complete [37]. The latter of these motivating factors indicates that any efficient quantum calculation, of fields or otherwise, can be transformed with polynomial resources to a scattering process of the interacting scalar field through the manipulation of classical external sources. As such, the entanglement structures found in the dynamical interacting scalar field are expected to be sufficient for the hardware implementation of efficient quantum computations. Perhaps surprisingly considering the naïve simplicity of the massless scalar field, an analytic calculation through conformal field theory of the entanglement structure between disjoint subregions even within one spatial dimension remains elusive, complicated in part by its spectroscopic nature with respect to field correlators. However, progress has developed through formidable analytic and high-precision numerical investigations of the entanglement structure of the scalar field both in the continuum and using a spatial lattice (harmonic chains) [38-50].

In the following, numerical results in the two-dimensional scalar field will be used to motivate discussions related to lattice quantum chromodynamics (LQCD) calculations 
[51-55] and low-energy effective field theories (EFTs) describing nuclear forces and other confining strongly interacting theories. Additional calculations in three dimensions are required to make quantitative predictions for previously unknown nonperturbative systematic errors in LQCD calculations, e.g., Refs. [56-59], and for potential impacts in nuclear EFTs, e.g., Refs. [60-64].

In order to quantify entanglement, the logarithmic negativity [65-68] between spatially separated regions of the field is calculated. The negativity - the sum of negative eigenvalues in the partially transposed reduced density matrix-quantifies violation of the parity symmetry in conjugate momentum space that would otherwise be exact in a tensor product state [68]. By quantifying the violation of a symmetry that would be exact for unentangled states, the negativity quantifies a deviation from separability. As a necessary but insufficient separability criterion, the negativity does not capture all quantum correlations [69], though it does provide an upper bound ${ }^{1}$ to the distillable entanglement $[67,72]$.

It has previously been observed that the negativity between individual oscillators of the latticized position-space scalar field vanish beyond nearest neighbor [39-42,44, 46-50,73]. This phenomenon is analogous to entanglement sudden death or early-stage disentanglement observed in the presence of quantum noise [74-77], where tracing of the scalar lattice external to the regions of interest provides the mechanism of decoherence. While the individual field operators $\hat{\phi}$ and $\hat{\pi}$ do not produce entanglement at long distances, individual creation and annihilation operators for position-space oscillators are sensitive to entanglement at long distances. The translation between these two bases involves a smearing in the field conjugate momentum space and points to the importance of such systematic delocalization for reproducing infrared entanglement properties through a lattice regularization. Naturally, higher resolution of physically separated field regions through a smaller lattice spacing improves agreement with continuum symmetries [78]. Though expected to systematically remove artifacts associated with finite lattice spacing, it is here found that finite negativity spheres are not perturbatively removed or expanded through Symanzik improvement [79] of the lattice dispersion relation. Supporting entangled regions of a latticized field demands a minimum information complexity of the field representation in the regions of interest, with a lattice spacing threshold only below which negativity spheres of sufficient size and accuracy are supported.

Consider circular regions of a 2D massless scalar field discretized onto a lattice (see Fig. 1). In the following, the negativity between the field degrees of freedom within such

\footnotetext{
${ }^{1}$ As an upper bound, the exponentially decaying negativity calculated in the continuum massless scalar field does not preclude the possibility that the distillable entanglement of the field is zero. Calculations harvesting entanglement from the scalar field suggest this is not the case $[10,70,71]$.
}

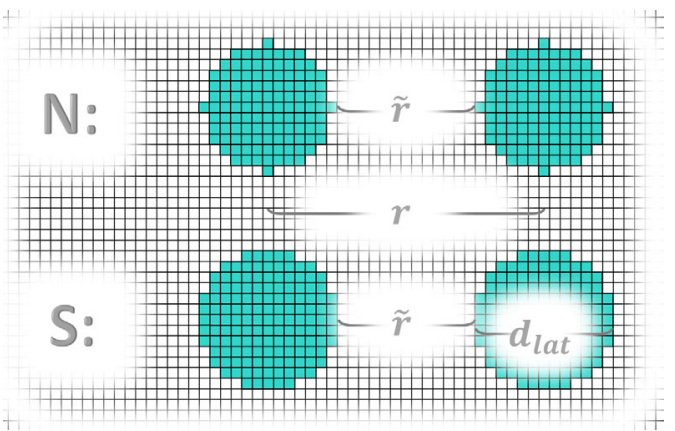

FIG. 1. Two choices of pixelation producing circular regions in the continuum.

regions with increased spatial separation is quantified. As conformal theories are without an intrinsic length scale, allowing calculations to be organized by relative dimensions in the continuum, all separations can be expressed in units of the region diameters, e.g., $\tilde{r} / d$. The tilde will be used to indicate a measurement of separation between the region surfaces rather than the distance between region centers. The choice of region diameter as a reference scale allows for a natural transition when considering the nucleon radius to set the scale for the field regions of interest in calculations of QCD. At finite lattice spacing, the continuum limit can be approached in an arbitrary number of ways. Two different pixelations of the regions are shown in Fig. 1. The method labeled " $N$ " begins with a central scalar field site and incorporates all sites within a specified integer radial distance. Organizing these sites into groups by the magnitude of their vector of center displacement integers, $|\mathbf{n}|^{2}$ [80], the $N$ boundary is defined with an integer truncation of $|\mathbf{n}|$. The method labeled " $S$ " incorporates additional $|\mathbf{n}|^{2}$ shells, truncating $|\mathbf{n}|$ at the next half integer. While the $S$ boundary approaches the continuum more rapidly, the independent perspectives provided by these two trajectories toward the continuum are found to be a valuable quantifier of systematic uncertainties. Calculating the negativity between these field regions determines a fundamental property of the field, the distillable entanglement present within the vacuum. A further application of this information to the operational feasibility of harvesting the present entanglement requires defining a detector structure and coupling to the studied field regions.

For a free noninteracting scalar field, all observables are expressible in terms of two-point vacuum expectation values of the field, $\hat{\phi}$, and conjugate momentum, $\hat{\pi}$, operators. In a finite volume with spatial extent $L$ in each direction and a lattice spacing set equal to one, these two-point functions are

$\langle\hat{\chi}(\mathbf{0}) \hat{\chi}(\mathbf{r})\rangle=\sum_{\mathbf{p}} \frac{e^{i \mathbf{p} \cdot \mathbf{r}}}{2 L^{D}}\left(m^{2}+\sum_{i} 4 \sin ^{2}\left(\frac{p_{i}}{2}\right)\right)^{\alpha / 2}$,

where $\alpha=1$ for $\hat{\chi}=\hat{\pi}, \alpha=-1$ for $\hat{\chi}=\hat{\phi}$, and $\mathbf{r}$ is a vector of integers. The discrete vector sum over momentum modes 

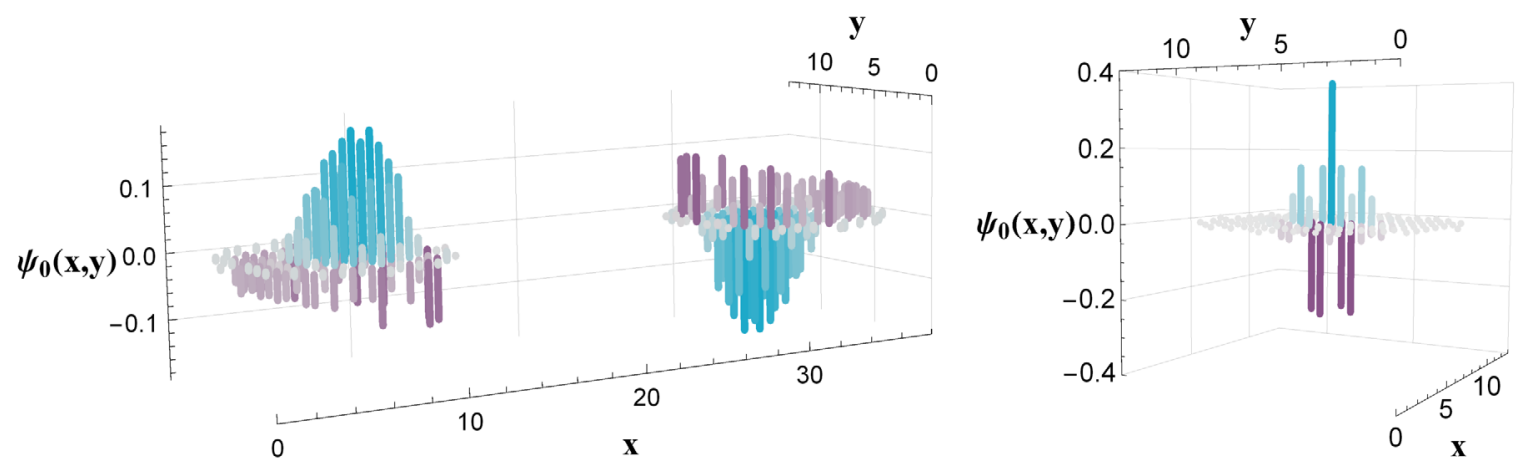

FIG. 2. Left: the ground-state wave function of the operator product $G H^{\Gamma}$ isolated to circular regions of $d_{\text {lat }}=13$ lattice sites across with the $N$-type boundary shown in Fig. 1 . Right: the ground-state wave function of one isolated region calculated with $\tilde{r}=100$, a separation beyond the negativity sphere, $\tilde{r}_{\mathcal{X}}$. Depicted numerical values can be found in Tables I and II of the Appendix.

incorporates each spatial component taking values in the set $p_{i} \epsilon \frac{2 \pi}{L} \mathbb{Z}_{L}$ with $\mathbb{Z}_{L}$ a bounded set of integers between 0 and $(L-1)$. While it is possible to calculate entanglement properties between two separated regions through analytic Gaussian integration of the field outside the regions to generate a reduced density operator [38,73], it is advantageous to instead use expectation values in the thermodynamic limit, $L \rightarrow \infty$, and properties of the symplectic spectrum to represent the calculation of entanglement with only propagators within and between the two regions $[42,81]$. Because the partial trace of a Gaussian state is implemented in the symplectic formalism as extracting diagonal covariance submatrices associated with the regions of interest, this choice allows efficient region isolation by eliminating intermediate computational steps requiring representation of the full lattice volume. In this case, the logarithmic negativity can be written as

$$
\mathcal{N}=-\sum_{i} \log _{2} \min \left(2 \sqrt{\lambda_{i}}, 1\right),
$$

where the $\lambda_{i}$ are eigenvalues of the matrix product $G H^{\Gamma}$ with $G_{x y}=\langle\hat{\phi}(\mathbf{x}) \hat{\phi}(\mathbf{y})\rangle, H_{x y}=\langle\hat{\pi}(\mathbf{x}) \hat{\pi}(\mathbf{y})\rangle$, and $\Gamma$ indicates the partial transposition of $H$. The coordinates comprising the basis of $G_{x y}$ and $H_{x y}$ are all sites within the two regions, requiring calculation of all two-point functions both within and between the regions of interest. Though not Hermitian, the product $G H^{\Gamma}$ enjoys real eigenvalues associated with the symmetric positive definiteness of $G$ and $H^{\Gamma}$. For interacting theories, in which higher-body correlation functions carry distinct information, this Gaussian approximation calculated from propagators alone is expected to provide a lower bound on the logarithmic negativity of the field [82]. For this continuous variable system, the partial transposition of $H$ can be implemented with a reflection in conjugate momentum space of the second region [68], $\pi(\mathbf{x}) \rightarrow-\pi(\mathbf{x})$ if $\mathbf{x}$ is the coordinate of a site within the second region. In practice, this partial transposition negates matrix elements of $H$ in the off-diagonal subblocks of propagators connecting the two regions. In the infinite volume limit (and continuous momentum within the first Brillouin zone) of twodimensional space, the two-point correlation functions populating $G$ and $H$ can be simplified to

$$
\begin{aligned}
\langle\hat{\chi}(\mathbf{0}) \hat{\chi}(\mathbf{r})\rangle= & \int_{0}^{\pi} \mathrm{d} p \frac{(6-2 \cos p)^{\alpha / 2} \cos \left(p r_{y}\right)}{2 \pi} \\
& \times{ }_{3} \tilde{F}_{2}\left(\begin{array}{l}
-\alpha / 2,1 / 2,1 \\
1-r_{x}, 1+r_{x} ; \frac{2}{3-\cos p}
\end{array}\right),
\end{aligned}
$$

where ${ }_{3} \tilde{F}_{2}$ is the regularized hypergeometric function. ${ }^{2}$ No infrared regulation is required in two dimensions, allowing the mass to be set to zero. ${ }^{3}$ In this formulation, with oscillatory integrands of increasing frequency and exponentially decreasing eigenvalues of the product $G H^{\Gamma}$ in the separation, along with increasing dimensionality of $G$ and $H$ in the lattice spacing, the calculation of the negativity exhibits a sign problem. As such, high precision is required (typically quadruple precision or greater) for evaluations of the $G, H$ integrals and the following eigenvalue determination, limiting the granularity of achievable region pixelations.

While the point-to-point propagators can be used directly as a basis for $G$ and $H$, it is convenient to form combinations that reflect the underlying symmetry of the pixelated regions: (i) the reflection symmetry in the plane along their separation axes and (ii) the perpendicular reflection plane at the midpoint of their separation. This leads to a block diagonalization of $G H^{\Gamma}$ into the symmetry sectors of the parity operators (which remain dense matrices). The negativity is dominated (by orders of magnitude at modest separation) by the lowest eigenvalue in the sector of $(+,-)$

\footnotetext{
${ }^{2}$ Note that a closed form expression exists for these two-point correlation functions.

${ }^{3}$ Massive theories will exhibit additional exponential suppression of the negativity controlled by the mass of the lightest particle. The massless limit has been chosen to isolate the purely geometric contribution.
} 

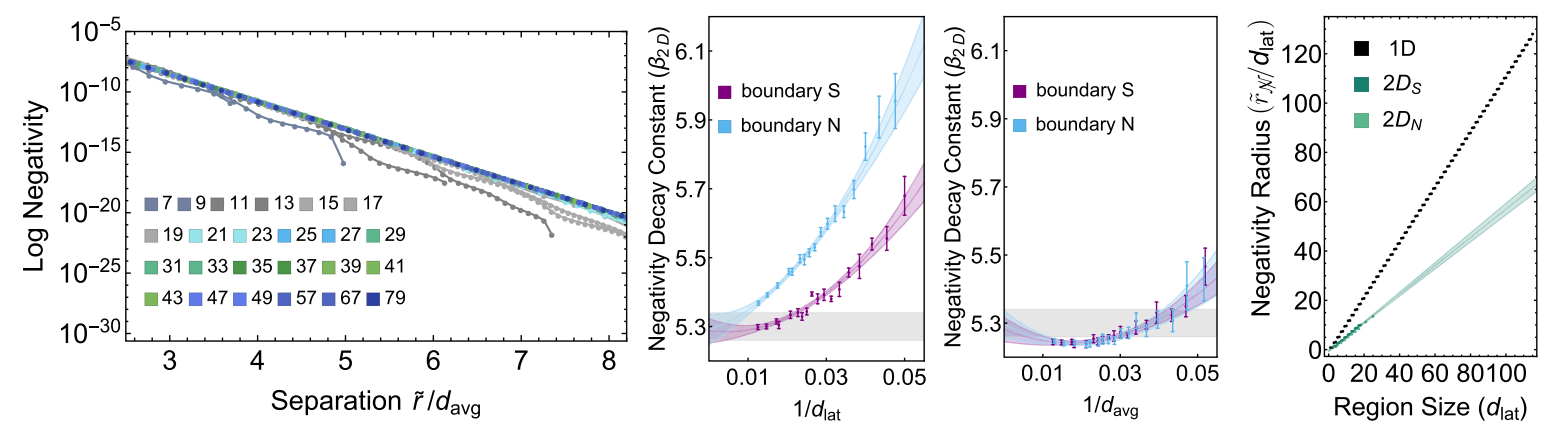

FIG. 3. Left: logarithmic negativity of two circular regions of the 2D massless noninteracting scalar field as a function of separation distance measured in units of the region diameter (see Fig. 1). Trajectories that end abruptly are found to exhibit zero negativity beyond a finite separation. Middle: negativity decay constants, $\beta_{2 D}$, extracted from the decay of logarithmic negativity as a function of the inverse region diameter (lattice spacing in units of the region diameter) extrapolated to zero with two pixelations of the circular field regions. Right: entanglement sphere radius, $\tilde{r}_{\mathcal{X}}$, as a function of region size in $1 \mathrm{D}$ and $2 \mathrm{D}$. Depicted numerical values can be found in Tables III-XIII of the Appendix.

parity for reflection planes $(1,2)$ described above. The wave function of this ground state of the product $G H^{\Gamma}$ is shown in the left panel in Fig. 2 for configuration $d_{\text {lat }}=\tilde{r}=13$. At a separation equal to one region diameter, this configuration is within the negativity sphere. The wave function shows that amplitudes experience an attractive interaction between the two regions, suggestive of a "flux tube" between them. With respect to the irreps of the dihedral group $D_{4}$, expressing a valid symmetry for individual regions and thus for a region at infinite separation, it is found that isolated contributions to the negativity are organized in the hierarchy of $\left(E, A_{1}, B_{1}, A_{2}, B_{2}\right)$ with the $E$ sector dominant at modest separations beyond the detector size. At separations beyond the negativity spheres, this apparent flux tube is broken and the wave function of a region appears as in the right panel in Fig. 2, calculated for $\tilde{r}=100$. The local horizontal asymmetry of each spatial region in the ground-state wave function rapidly decays and the wave function acquires approximately an oscillatory Gaussian envelope. In the continuum limit at infinite separation, these regions carry zero "charge."4

The results of computing the logarithmic negativity between these circular regions are shown in the left panel in Fig. 3. In the continuum limit, the logarithmic negativity decay is dominated by an exponential with the separation measured in units of the region size, $\tilde{r} / d$, where $d$ is the diameter across the region and $\tilde{r}$ is the separation between the regions, $\mathcal{N} \sim r^{\alpha} e^{-\beta(\tilde{r} / d)}$. Considering only the exponential behavior and not the subleading power law, ${ }^{5}$ this behavior is controlled by a pure number extrapolated in the second and third panels in Fig. 3 to be $\beta_{2 D}=5.29(4)$ in two dimensions. In the second panel, the reference length

\footnotetext{
${ }^{4}$ The sum of the amplitudes in the wave function of each isolated region vanishes in the continuum.

${ }^{5}$ The complete functional form will be required for future quantitative estimates of systematic uncertainties in both classical and quantum simulations.
}

scale associated with the regions, $d$, was chosen to be the largest extent of the circular region along the lattice axis (for the example regions of Fig. 1, $d_{\text {lat }}=13$ ). This classification places the $S$ and $N$ boundary structures on different trajectories toward the continuum limit of rotationally symmetric field regions. In the third panel in Fig. 3, the reference length scale associated with the field region was chosen to be an averaged diameter calculated from each point on the boundary (for the $d_{\text {lat }}=13$ example regions of Fig. 1, the $S$ boundary has $d_{\text {avg }}=12.36$ while the $N$ boundary has $d_{\text {avg }}=11.27$ ). This classification connects the trajectories of the $S$ and $N$ boundary structures. The extrapolated entanglement mass $\beta_{2 D}$ is consistent between these two diameter definitions, though the latter is found to produce negativity within $1 \%$ of the continuum value at larger lattice spacing, as expected for a continuuminspired spatial averaging. The resulting value of $\beta_{2 D}$ is distinct from that previously calculated in one dimension, $\beta_{1 D}=2.82(3)$ [42], indicating a more rapid decay of distillable entanglement within the massless scalar vacuum in higher-dimensional space. It is expected, and the subject of future work, that $\beta_{3 D}$ will be further suppressed.

The existence of a pure number $\beta$ in the massless noninteracting scalar field acting to exponentially suppress quantum correlations in the continuum, as would the presence of a mass scale, presents an opportunity for the appearance of an additional relative scale associated with the geometric entanglement structure in systems without conformal symmetry. With this mechanism, the previous observation of an entanglement-based hierarchy in lowenergy nuclear interactions [27], normally predicated on the dominance of local operators, could be understood even without an explicit dimensionful parameter quantifying the entanglement. For example, expecting $\beta_{3 D}$ to be found of similar magnitude to $\beta_{2 D}$, a rescaling of the radius of the nucleon set by the pion mass with a factor of $\beta$ empirically produces a mass scale of $\sim 350 \mathrm{MeV}$, a scale found to characterize the convergence of EFT descriptions of 
nucleon-nucleon interactions when accounting for the characteristic and unjustifiably large scattering lengths [63]. Alternatively, a naïve expectation that $\beta_{3 D} \sim 7.7$, approximately linearly extrapolated from $\beta_{1 D, 2 D}$, could indicate an entanglement scale associated with the pion mass to be found around the scale of chiral symmetry breaking. As expected, the numerical results of the twodimensional massless scalar field are not sufficient to conclusively determine the role played by the negativity decay constant $\beta$ in the design of low-energy effective field theories of the strong interactions. However, these preliminary considerations motivate the nonperturbative computation of $\beta_{3 D}$ in both the scalar field and QCD, expected to require significant HPC (high-performance computing) resources.

Consistent with an understanding that the lattice representation preferentially captures local properties of the field structure and nonlocal properties only in the $a \rightarrow 0$ limit, calculations of the negativity at finite lattice spacing nonperturbatively vanish beyond a particular radius $\tilde{r}_{\mathcal{X}}$. The right panel in Fig. 3 shows the radius of the sphere supporting nonzero negativity, $\tilde{r}_{\mathcal{X}}$, measured in units of the spatial extent of the entangled regions for a $1 \mathrm{D}$ and 2D lattice. It is clear that the entanglement radius grows more slowly on a 2D lattice as $\tilde{r}_{\mathcal{X}} / d_{\text {lat }} \sim 0.59(4) d_{\text {lat }}$ compared with that in $1 \mathrm{D}, \tilde{r}_{X} / d_{\text {lat }} \sim 1.118(1) d_{\text {lat }}$. The further constricted negativity sphere radius expected to be found in a 3D geometry warrants exploration of this nonperturbative lattice effect for reliable calculation of coherent quantum phenomena on both classical and quantum computational frameworks.

A nonvanishing lattice spacing introduces features beyond the finite radius negativity sphere. The negativity exhibits an oscillatory component with an amplitude that vanishes in the continuum limit and falls away from the continuum value as it approaches $\tilde{r}_{\mathcal{X}}$, the surface of the negativity sphere. These oscillations in negativity introduce an additional systematic error in lattice calculations to be considered, even within $\tilde{r}_{x}$. For small systems, this can lead to orders of magnitude deviations in the negativity from the continuum limit.

The finite value of $\tilde{r}_{\mathcal{X}}$ implies the existence of a nonperturbative reduction in the physical entanglement volume of a lattice calculation if the observable of interest is sensitive to distillable entanglement. In order to begin understanding the potential implications of the latticespacing-induced finite-sized negativity sphere for LQCD calculations, we consider relevant lengths scales in a $2 \mathrm{D}$ lattice calculation of two "nucleon-sized" objects interacting through a massless scalar field.

Assuming the nucleon radius is defined by the QCD chiral symmetry-breaking scale $r \chi \sim 1 / \Lambda_{\chi} \sim 0.2 \mathrm{fm}$, and the scalar field is defined on a $2 \mathrm{D}$ grid with a lattice spacing of $a \sim 0.1 \mathrm{fm}$ (corresponding to $d_{\chi} \sim 5$ lattice sites across the nucleon), the radius of the negativity sphere is
$\tilde{r}_{\mathcal{X}} \sim 0.8 \mathrm{fm}$. At this radius, the logarithmic negativity is $\mathcal{N} \sim 10^{-7}$. Therefore, beyond a separation of $r_{\mathcal{X}} \sim 1.2 \mathrm{fm}$, the long-distance entanglement structure of the system is incorrect but only at the level of $\lesssim 10^{-7}$ in the distillable entanglement. A slightly increased lattice spacing of $a=0.15 \mathrm{fm}$ corresponds to a vanishing of the logarithmic negativity at $\tilde{r}_{X} \sim 0.3 \mathrm{fm}$, introducing an entanglement error at the $10^{-5}$ level. If the size of the nucleons is set by the physical pion mass, $r \chi \sim 1 / m_{\pi} \sim 1.4 \mathrm{fm}$ (corresponding to $d_{m_{\pi}} \sim 30$ lattice sites across for $a=0.1 \mathrm{fm}$ ), the negativity sphere has a radius of $\tilde{r}_{\mathcal{X}} \sim 47 \mathrm{fm}$ with $\mathcal{N} \sim 1 \times 10^{-40}$. In the case of lattice EFT [83-86] with dynamical pions, the death of entanglement is likely of greater significance as lattice spacings tend to be larger than those applied in the estimates above.

These 2D estimates indicate that, for coherent quantum observables, LQCD calculations with coarse lattice spacings and quark masses that are physical or heavier may vary in their reliability, with these errors exponentially shrinking with lattice spacing or region pixelation. Translating the above observations to LQCD and lattice EFT calculations can only be at a qualitative level without further, in situ, numerical investigations in 3D. For LQCD calculations, a much more complex set of estimates are required as the size of the nucleon is dominated by its coupling to pions, which are excitations of the quark condensate, that behave like a fundamental pseudoscalar field only at low energies.

However, many classical observables are likely to be insensitive to a lattice truncation of the negativity, as suggested by smooth two-point functions of the field operators and $(1 \times 1)$-site mutual information calculated in a massive scalar field, reflecting continuum structure with only nearest neighbor $(1 \times 1)$-site negativity [39-42, $44,46,48,49,73]$. This perspective aligns well with the successes of semiclassical approximations to the structure and interactions of nuclei, including the large- $N_{c}$ limit of QCD [87-92]. Propagating the impact of the nonperturbative negativity sphere to provide a complete quantification of uncertainties for specifically quantum observables requires further research.

Beyond the two-body negativity spheres, we anticipate irreducible three-body negativity spheres involving three spatially separated regions that do not factorize into combinations of one-body and two-body negativities. While such irreducibility is well established for qubit systems [93-96], similar quantities remain to be defined in continuous systems of spatially extended field regions.

In LQCD calculations, Luscher's methods [97-99] have played a central role in extracting physics from finitevolume, Euclidean-space computations. These methods are applicable to simulations on quantum devices with little or no modification and are expected to play an important role for near-term simulations in small spatial volumes. Finite lattice spacing artifacts are treated as distinct in LQCD calculations, as they are UV effects, while Luscher's 
methods are related to the IR structure of the simulation volume. The impact of the lattice-induced negativity sphere $\tilde{r}_{x}$ on finite-volume wave functions remains to be assessed, though expected to be small for all but quantum coherent observables.

The implications of negativity spheres $\tilde{r}_{X}$ in the context of EFTs is interesting to consider further. At the heart of such effective descriptions are multipole expansions. This framework enables the effects of extended sources and sinks in quantum field theories (QFTs) to be included in low-energy EFTs as local operators $\left[\delta^{(n)}(\mathbf{r}), \delta^{(n)}(\mathbf{r}), \ldots\right]$ coupled to the dynamical IR degrees of freedom as in, for example, heavy-baryon chiral perturbation theory [100]. As EFTs require both an operator structure and a prescribed regularization and renormalization scheme, extending the effects of the negativity sphere to the EFT suggests that taking renormalization scales that are disparate from the "size" of the source or sink could lead to discrepancies in the low-energy description of entanglement. This point may be relevant to EFT descriptions of baryons [100] and of nuclear forces [60-64], particularly nucleon-nucleon interactions in channels with a tensor force, which have so far eluded dimensional regularization [101-103] but can be regulated with smearing in either momentum or position space. In the case of single baryons, it has been suggested that a spatial regularization would have advantages over massless regularization schemes in the convergence of chiral expansions of baryon properties [104].

In addition to the implications of geometrically influenced entanglement scales in EFT construction and of negativity spheres in designing latticized calculations of quantum fields, a perspective remains that detailed understanding of UV and IR entanglement properties can be leveraged for computational advantage [73,105-108]. While the IR physics of interest may be insensitive to specific choices in the UV structure, demands on computational hardware remain susceptible. The finite negativity sphere at distance $\tilde{r}_{x}$ scaling inversely with the pixelation of the field region indicates that UV operators (probing length scales of the lattice spacing) cannot access entangled elements of the field at long distances. It is possible that this delocalization may be leveraged to improve robustness of quantum hardware against locally interacting, classically correlated sources of noise when simulating QFTs. Alternatively, designing a field representation with UV entanglement mirroring that found in the IR may provide a reduction in lattice artifacts for coherent quantum observables.

Exploring systematic improvement of the dispersion relation $2 \sin \frac{p_{i}}{2} \rightarrow p_{i}$ reveals that the radius of the lattice negativity sphere $\tilde{r}_{X}$ and the geometric decay constant $\beta$ are essentially unchanged. Operator and field smearing plays a key role in LQCD calculations, tempering UV fluctuations enabling convergence for low-energy quantities and mitigating the impact of $\mathrm{SO}(3)$ breaking due to the $\mathrm{H}(3)$ spatial lattice. We have not performed an extensive study of the impact of field or operator smearing, beyond the dispersion relation, on quantum coherence.

The 2D numerical results provided in this work indicate that the bound on distillable entanglement between two spatially separated regions of the massless noninteracting scalar field vacuum is defined by a decay constant increasing with the dimensionality of spacetime. Viewed as preliminary evidence of similar properties in more complex gauge theories-such as QCD in which a composite (pseudo)scalar field mediates the long-distance interaction between nucleons - the potential impact of this geometric decay constant in providing an entanglement-sensitive scale in the EFT description of nuclei is discussed. When pixelating the regions of interest and latticizing the field for nonperturbative calculation, the distillable entanglement is found to suddenly vanish at geometrically large separations (relative to the region size) again dependent on the spatial dimension, becoming more artificially localized in higher dimension. A thorough and quantitative understanding of the lattice-induced truncation of the distillable entanglement, from the scalar field to QCD, will be a necessary foundation for the reliable calculation of entangled field excitations as well as their propagation to large distances, e.g., when probing real-time coherent fragmentation processes, a central target for quantum simulation. With reduced lattice spacing providing the main source of improvement, the complexity of manybody interactions between collections of lattice sites is determined to be essential for supporting quantum phenomena. The implications of these geometric features of entanglement in quantum fields, on the convergence of low-energy EFTs and the regulation of spatially extended field objects, sheds new light on objectives to nonperturbatively express nonlocal quantum effects through a hierarchy of local operators and field elements.

\section{ACKNOWLEDGMENTS}

We would like to thank Silas Beane, Ramya Bhaskar, Joe Carlson, David Kaplan, Pavel Lougovski, Aidan Murran, Caroline Robin, Kenneth Roche, and Alessandro Roggero for valuable discussions. We would also like to thank Center for Experimental Nuclear Physics and Astrophysics (CENPA) at the University of Washington for providing an effective work environment over a period of many months for processing and developing many of the ideas and calculations presented in this paper. Some of this work was performed on the UW's HYAK High Performance and Data Ecosystem. We have made extensive use of Wolfram Mathematica [109] and the Avanpix multiprecision computing toolbox [110] for MATLAB [111]. N. K. and M. J. S. were supported in part by DOE Grant No. DE-FG0200ER41132 and Fermi National Accelerator Laboratory PO No. 652197. This work is supported in part by the U.S. Department of Energy, Office of Science, Office of Advanced Scientific Computing Research (ASCR) 
quantum algorithm teams program, under field work Proposal No. ERKJ333. N. K. was supported in part by a Microsoft Research Ph.D. fellowship.

\section{APPENDIX: TABLES}

TABLE I. Wave function amplitudes shown in the left panel in Fig. 2.

\begin{tabular}{|c|c|c|}
\hline$x$ & $y$ & $\psi_{0}(x, y)$ \\
\hline 6 & 0 & 0.0053146 \\
\hline 3 & 1 & 0.0060582 \\
\hline 4 & 1 & 0.0068117 \\
\hline 5 & 1 & 0.010010 \\
\hline 6 & 1 & -0.077618 \\
\hline 7 & 1 & 0.0072781 \\
\hline 8 & 1 & 0.0042535 \\
\hline 9 & 1 & 0.0044180 \\
\hline 2 & 2 & 0.0082886 \\
\hline 3 & 2 & -0.043739 \\
\hline 4 & 2 & -0.050578 \\
\hline 5 & 2 & -0.040683 \\
\hline 6 & 2 & -0.043948 \\
\hline 7 & 2 & -0.031974 \\
\hline 8 & 2 & -0.052302 \\
\hline 9 & 2 & -0.089283 \\
\hline 10 & 2 & 0.013301 \\
\hline 1 & 3 & 0.0057025 \\
\hline 2 & 3 & -0.034449 \\
\hline 3 & 3 & -0.046326 \\
\hline 4 & 3 & -0.027783 \\
\hline 5 & 3 & 0.0019202 \\
\hline 6 & 3 & 0.026263 \\
\hline 7 & 3 & 0.039338 \\
\hline 8 & 3 & 0.022905 \\
\hline 9 & 3 & -0.028781 \\
\hline 10 & 3 & -0.089620 \\
\hline 11 & 3 & 0.0012799 \\
\hline 1 & 4 & 0.0067524 \\
\hline 2 & 4 & -0.041270 \\
\hline 3 & 4 & -0.030045 \\
\hline 4 & 4 & 0.0076432 \\
\hline 5 & 4 & 0.053102 \\
\hline 6 & 4 & 0.092581 \\
\hline 7 & 4 & 0.11486 \\
\hline 8 & 4 & 0.10603 \\
\hline 9 & 4 & 0.055241 \\
\hline 10 & 4 & -0.029216 \\
\hline 11 & 4 & -0.0054380 \\
\hline 1 & 5 & 0.0068186 \\
\hline 2 & 5 & -0.038103 \\
\hline 3 & 5 & -0.014644 \\
\hline 4 & 5 & 0.034162 \\
\hline 5 & 5 & 0.089135 \\
\hline 6 & 5 & 0.13717 \\
\hline 7 & 5 & 0.16628 \\
\hline
\end{tabular}

TABLE I. (Continued)

\begin{tabular}{|c|c|c|}
\hline$x$ & $y$ & $\psi_{0}(x, y)$ \\
\hline 8 & 5 & 0.16310 \\
\hline 9 & 5 & 0.11490 \\
\hline 10 & 5 & 0.023216 \\
\hline 11 & 5 & 0.0027471 \\
\hline 0 & 6 & 0.0049114 \\
\hline 1 & 6 & -0.036499 \\
\hline 2 & 6 & -0.043229 \\
\hline 3 & 6 & -0.0099229 \\
\hline 4 & 6 & 0.043510 \\
\hline 5 & 6 & 0.10181 \\
\hline 6 & 6 & 0.15268 \\
\hline 7 & 6 & 0.18416 \\
\hline 8 & 6 & 0.18274 \\
\hline 9 & 6 & 0.13402 \\
\hline 10 & 6 & 0.029073 \\
\hline 11 & 6 & -0.10248 \\
\hline 12 & 6 & -0.0037411 \\
\hline 1 & 7 & 0.0068186 \\
\hline 2 & 7 & -0.038103 \\
\hline 3 & 7 & -0.014644 \\
\hline 4 & 7 & 0.034162 \\
\hline 5 & 7 & 0.089135 \\
\hline 6 & 7 & 0.13717 \\
\hline 7 & 7 & 0.16628 \\
\hline 8 & 7 & 0.16310 \\
\hline 9 & 7 & 0.11490 \\
\hline 10 & 7 & 0.023216 \\
\hline 11 & 7 & 0.0027471 \\
\hline 1 & 8 & 0.0067524 \\
\hline 2 & 8 & -0.041270 \\
\hline 3 & 8 & -0.030045 \\
\hline 4 & 8 & 0.0076432 \\
\hline 5 & 8 & 0.053102 \\
\hline 6 & 8 & 0.092581 \\
\hline 7 & 8 & 0.11486 \\
\hline 8 & 8 & 0.10603 \\
\hline 9 & 8 & 0.055241 \\
\hline 10 & 8 & -0.029216 \\
\hline 11 & 8 & -0.0054380 \\
\hline 1 & 9 & 0.0057025 \\
\hline 2 & 9 & -0.034449 \\
\hline 3 & 9 & -0.046326 \\
\hline 4 & 9 & -0.027783 \\
\hline 5 & 9 & 0.0019202 \\
\hline 6 & 9 & 0.026263 \\
\hline 7 & 9 & 0.039338 \\
\hline 8 & 9 & 0.022905 \\
\hline 9 & 9 & -0.028781 \\
\hline 10 & 9 & -0.089620 \\
\hline 11 & 9 & 0.0012799 \\
\hline 2 & 10 & 0.0082886 \\
\hline 3 & 10 & -0.043739 \\
\hline 4 & 10 & -0.050578 \\
\hline 5 & 10 & -0.040683 \\
\hline 6 & 10 & -0.043948 \\
\hline 7 & 10 & -0.031974 \\
\hline
\end{tabular}


TABLE I. (Continued)

\begin{tabular}{|c|c|c|c|c|c|}
\hline$x$ & $y$ & $\psi_{0}(x, y)$ & $x$ & $y$ & $\psi_{0}(x, y)$ \\
\hline 8 & 10 & -0.052302 & 33 & 5 & -0.089135 \\
\hline 9 & 10 & -0.089283 & 34 & 5 & -0.034162 \\
\hline 10 & 10 & 0.013301 & 35 & 5 & 0.014644 \\
\hline 3 & 11 & 0.0060582 & 36 & 5 & 0.038103 \\
\hline 4 & 11 & 0.0068117 & 37 & 5 & -0.0068186 \\
\hline 5 & 11 & 0.010010 & 26 & 6 & 0.0037411 \\
\hline 6 & 11 & -0.077618 & 27 & 6 & 0.10248 \\
\hline 7 & 11 & 0.0072781 & 28 & 6 & -0.029073 \\
\hline 8 & 11 & 0.0042535 & 29 & 6 & -0.13402 \\
\hline 9 & 11 & 0.0044180 & 30 & 6 & -0.18274 \\
\hline 6 & 12 & 0.0053146 & 31 & 6 & -0.18416 \\
\hline 32 & 0 & -0.0053146 & 32 & 6 & -0.15268 \\
\hline 29 & 1 & -0.0044180 & 33 & 6 & -0.10181 \\
\hline 30 & 1 & -0.0042535 & 34 & 6 & -0.043510 \\
\hline 31 & 1 & -0.0072781 & 35 & 6 & 0.0099229 \\
\hline 32 & 1 & 0.077618 & 36 & 6 & 0.043229 \\
\hline 33 & 1 & -0.010010 & 37 & 6 & 0.036499 \\
\hline 34 & 1 & -0.0068117 & 38 & 6 & -0.0049114 \\
\hline 35 & 1 & -0.0060582 & 27 & 7 & -0.0027471 \\
\hline 28 & 2 & -0.013301 & 28 & 7 & -0.023216 \\
\hline 29 & 2 & 0.089283 & 29 & 7 & -0.11490 \\
\hline 30 & 2 & 0.052302 & 30 & 7 & -0.16310 \\
\hline 31 & 2 & 0.031974 & 31 & 7 & -0.16628 \\
\hline 32 & 2 & 0.043948 & 32 & 7 & -0.13717 \\
\hline 33 & 2 & 0.040683 & 33 & 7 & -0.089135 \\
\hline 34 & 2 & 0.050578 & 34 & 7 & -0.034162 \\
\hline 35 & 2 & 0.043739 & 35 & 7 & 0.014644 \\
\hline 36 & 2 & -0.0082886 & 36 & 7 & 0.038103 \\
\hline 27 & 3 & -0.0012799 & 37 & 7 & -0.0068186 \\
\hline 28 & 3 & 0.089620 & 27 & 8 & 0.0054380 \\
\hline 29 & 3 & 0.028781 & 28 & 8 & 0.029216 \\
\hline 30 & 3 & -0.022905 & 29 & 8 & -0.055241 \\
\hline 31 & 3 & -0.039338 & 30 & 8 & -0.10603 \\
\hline 32 & 3 & -0.026263 & 31 & 8 & -0.11486 \\
\hline 33 & 3 & -0.0019202 & 32 & 8 & -0.092581 \\
\hline 34 & 3 & 0.027783 & 33 & 8 & -0.053102 \\
\hline 35 & 3 & 0.046326 & 34 & 8 & -0.0076432 \\
\hline 36 & 3 & 0.034449 & 35 & 8 & 0.030045 \\
\hline 37 & 3 & -0.0057025 & 36 & 8 & 0.041270 \\
\hline 27 & 4 & 0.0054380 & 37 & 8 & -0.0067524 \\
\hline 28 & 4 & 0.029216 & 27 & 9 & -0.0012799 \\
\hline 29 & 4 & -0.055241 & 28 & 9 & 0.089620 \\
\hline 30 & 4 & -0.10603 & 29 & 9 & 0.028781 \\
\hline 31 & 4 & -0.11486 & 30 & 9 & -0.022905 \\
\hline 32 & 4 & -0.092581 & 31 & 9 & -0.039338 \\
\hline 33 & 4 & -0.053102 & 32 & 9 & -0.026263 \\
\hline 34 & 4 & -0.0076432 & 33 & 9 & -0.0019202 \\
\hline 35 & 4 & 0.030045 & 34 & 9 & 0.027783 \\
\hline 36 & 4 & 0.041270 & 35 & 9 & 0.046326 \\
\hline 37 & 4 & -0.0067524 & 36 & 9 & 0.034449 \\
\hline 27 & 5 & -0.0027471 & 37 & 9 & -0.0057025 \\
\hline 28 & 5 & -0.023216 & 28 & 10 & -0.013301 \\
\hline 29 & 5 & -0.11490 & 29 & 10 & 0.089283 \\
\hline 30 & 5 & -0.16310 & 30 & 10 & 0.052302 \\
\hline 31 & 5 & -0.16628 & 31 & 10 & 0.031974 \\
\hline 32 & 5 & -0.13717 & 32 & 10 & 0.043948 \\
\hline
\end{tabular}

TABLE I. (Continued)

TABLE I. (Continued)


TABLE I. (Continued)

\begin{tabular}{lcr}
\hline \hline$x$ & $y$ & $\psi_{0}(x, y)$ \\
\hline 33 & 10 & 0.040683 \\
34 & 10 & 0.050578 \\
35 & 10 & 0.043739 \\
36 & 10 & -0.0082886 \\
29 & 11 & -0.0044180 \\
30 & 11 & -0.0042535 \\
31 & 11 & -0.0072781 \\
32 & 11 & 0.077618 \\
33 & 11 & -0.010010 \\
34 & 11 & -0.0068117 \\
35 & 11 & -0.0060582 \\
32 & 12 & -0.0053146 \\
\hline \hline
\end{tabular}

TABLE II. Wave function amplitudes shown in the right panel in Fig. 2.

\begin{tabular}{|c|c|c|}
\hline$x$ & $y$ & $\psi_{0}(x, y)$ \\
\hline 6 & 0 & $4.9034 \times 10^{-8}$ \\
\hline 3 & 1 & $-5.2388 \times 10^{-9}$ \\
\hline 4 & 1 & $-8.1054 \times 10^{-8}$ \\
\hline 5 & 1 & $-1.3491 \times 10^{-6}$ \\
\hline 6 & 1 & $6.5912 \times 10^{-6}$ \\
\hline 7 & 1 & $-1.3237 \times 10^{-6}$ \\
\hline 8 & 1 & $-6.7897 \times 10^{-8}$ \\
\hline 9 & 1 & $-3.8544 \times 10^{-9}$ \\
\hline 2 & 2 & $-8.6614 \times 10^{-9}$ \\
\hline 3 & 2 & $-2.7111 \times 10^{-7}$ \\
\hline 4 & 2 & $-8.1358 \times 10^{-6}$ \\
\hline 5 & 2 & 0.00019040 \\
\hline 6 & 2 & -0.00051386 \\
\hline 7 & 2 & 0.00018701 \\
\hline 8 & 2 & $-8.9529 \times 10^{-6}$ \\
\hline 9 & 2 & $-2.5605 \times 10^{-7}$ \\
\hline 10 & 2 & $-5.1844 \times 10^{-9}$ \\
\hline 1 & 3 & $-5.3318 \times 10^{-9}$ \\
\hline 2 & 3 & $-2.7117 \times 10^{-7}$ \\
\hline 3 & 3 & -0.000013289 \\
\hline 4 & 3 & 0.00061389 \\
\hline 5 & 3 & -0.0045493 \\
\hline 6 & 3 & 0.0092643 \\
\hline 7 & 3 & -0.0044675 \\
\hline 8 & 3 & 0.00061098 \\
\hline 9 & 3 & -0.000015291 \\
\hline 10 & 3 & $-2.5654 \times 10^{-7}$ \\
\hline 11 & 3 & $-3.1143 \times 10^{-9}$ \\
\hline 1 & 4 & $-8.2163 \times 10^{-8}$ \\
\hline 2 & 4 & $-8.0923 \times 10^{-6}$ \\
\hline 3 & 4 & 0.00061368 \\
\hline 4 & 4 & -0.0083757 \\
\hline
\end{tabular}

TABLE II. (Continued)

\begin{tabular}{|c|c|c|}
\hline$x$ & $y$ & $\psi_{0}(x, y)$ \\
\hline 5 & 4 & 0.039844 \\
\hline 6 & 4 & -0.069559 \\
\hline 7 & 4 & 0.039077 \\
\hline 8 & 4 & -0.0081646 \\
\hline 9 & 4 & 0.00060715 \\
\hline 10 & 4 & $-9.2898 \times 10^{-6}$ \\
\hline 11 & 4 & $-5.7750 \times 10^{-8}$ \\
\hline 1 & 5 & $-1.3578 \times 10^{-6}$ \\
\hline 2 & 5 & 0.00019088 \\
\hline 3 & 5 & -0.0045545 \\
\hline 4 & 5 & 0.039877 \\
\hline 5 & 5 & -0.15121 \\
\hline 6 & 5 & 0.24273 \\
\hline 7 & 5 & -0.14799 \\
\hline 8 & 5 & 0.038412 \\
\hline 9 & 5 & -0.0043252 \\
\hline 10 & 5 & 0.00017830 \\
\hline 11 & 5 & $-1.2394 \times 10^{-6}$ \\
\hline 0 & 6 & $4.4273 \times 10^{-8}$ \\
\hline 1 & 6 & $7.0459 \times 10^{-6}$ \\
\hline 2 & 6 & -0.00052161 \\
\hline 3 & 6 & 0.0093308 \\
\hline 4 & 6 & -0.069963 \\
\hline 5 & 6 & 0.24398 \\
\hline 6 & 6 & -0.37969 \\
\hline 7 & 6 & 0.23827 \\
\hline 8 & 6 & -0.066852 \\
\hline 9 & 6 & 0.0086482 \\
\hline 10 & 6 & -0.00045085 \\
\hline 11 & 6 & $4.0623 \times 10^{-6}$ \\
\hline 12 & 6 & $6.6846 \times 10^{-8}$ \\
\hline 1 & 7 & $-1.3578 \times 10^{-6}$ \\
\hline 2 & 7 & 0.00019088 \\
\hline 3 & 7 & -0.0045545 \\
\hline 4 & 7 & 0.039877 \\
\hline 5 & 7 & -0.15121 \\
\hline 6 & 7 & 0.24273 \\
\hline 7 & 7 & -0.14799 \\
\hline 8 & 7 & 0.038412 \\
\hline 9 & 7 & -0.0043252 \\
\hline 10 & 7 & 0.00017830 \\
\hline 11 & 7 & $-1.2394 \times 10^{-6}$ \\
\hline 1 & 8 & $-8.2163 \times 10^{-8}$ \\
\hline 2 & 8 & $-8.0923 \times 10^{-6}$ \\
\hline 3 & 8 & 0.00061368 \\
\hline 4 & 8 & -0.0083757 \\
\hline 5 & 8 & 0.039844 \\
\hline 6 & 8 & -0.069559 \\
\hline 7 & 8 & 0.039077 \\
\hline 8 & 8 & -0.0081646 \\
\hline 9 & 8 & 0.00060715 \\
\hline 10 & 8 & $-9.2898 \times 10^{-6}$ \\
\hline 11 & 8 & $-5.7750 \times 10^{-8}$ \\
\hline 1 & 9 & $-5.3318 \times 10^{-9}$ \\
\hline 2 & 9 & $-2.7117 \times 10^{-7}$ \\
\hline
\end{tabular}


TABLE II. (Continued)

\begin{tabular}{lcc}
\hline \hline$x$ & $y$ & $\psi_{0}(x, y)$ \\
\hline 3 & 9 & -0.000013289 \\
4 & 9 & 0.00061389 \\
5 & 9 & -0.0045493 \\
6 & 9 & 0.0092643 \\
7 & 9 & -0.0044675 \\
8 & 9 & 0.00061098 \\
9 & 9 & -0.000015291 \\
10 & 9 & $-2.5654 \times 10^{-7}$ \\
11 & 9 & $-3.1143 \times 10^{-9}$ \\
2 & 10 & $-8.6614 \times 10^{-9}$ \\
3 & 10 & $-2.7111 \times 10^{-7}$ \\
4 & 10 & $-8.1358 \times 10^{-6}$ \\
5 & 10 & 0.00019040 \\
6 & 10 & -0.00051386 \\
7 & 10 & 0.00018701 \\
8 & 10 & $-8.9529 \times 10^{-6}$ \\
9 & 10 & $-2.5605 \times 10^{-7}$ \\
10 & 10 & $-5.1844 \times 10^{-9}$ \\
3 & 11 & $-5.2388 \times 10^{-9}$ \\
4 & 11 & $-8.1054 \times 10^{-8}$ \\
5 & 11 & $-1.3491 \times 10^{-6}$ \\
6 & 11 & $6.5912 \times 10^{-6}$ \\
7 & 11 & $-1.3237 \times 10^{-6}$ \\
8 & 11 & $-6.7897 \times 10^{-8}$ \\
9 & 11 & $-3.8544 \times 10^{-9}$ \\
6 & 12 & $4.9034 \times 10^{-8}$ \\
\hline \hline & & \\
\hline & & 9
\end{tabular}

TABLE III. Logarithmic negativity as a function of separation measured in units of the averaged region diameter with an $N$-type boundary appearing in the left panel in Fig. 3.

\begin{tabular}{lc}
\hline \hline & $d_{\text {lat }}=7$ \\
\hline$\tilde{r} / d_{\text {avg }}$ & $\mathcal{N}$ \\
\hline 0.553 & $1.20 \times 10^{-3}$ \\
0.737 & $5.90 \times 10^{-4}$ \\
0.922 & $2.58 \times 10^{-4}$ \\
1.11 & $9.09 \times 10^{-5}$ \\
1.29 & $2.29 \times 10^{-5}$ \\
1.47 & $6.81 \times 10^{-6}$ \\
1.66 & $2.91 \times 10^{-6}$ \\
1.84 & $1.32 \times 10^{-6}$ \\
2.03 & $5.12 \times 10^{-7}$ \\
2.21 & $1.57 \times 10^{-7}$ \\
2.4 & $4.84 \times 10^{-8}$ \\
2.58 & $1.29 \times 10^{-8}$ \\
2.77 & $2.41 \times 10^{-9}$ \\
2.95 & $7.82 \times 10^{-10}$ \\
\hline
\end{tabular}

TABLE III. (Continued)

\begin{tabular}{lcc}
\hline \hline & $d_{\text {lat }}=7$ & \\
\hline$\tilde{r} / d_{\text {avg }}$ & $\mathcal{N}$ \\
\hline 3.13 & & $3.66 \times 10^{-10}$ \\
3.32 & $1.83 \times 10^{-10}$ \\
3.5 & $8.01 \times 10^{-11}$ \\
3.69 & $1.32 \times 10^{-11}$ \\
\hline \hline & & \\
\hline
\end{tabular}

\begin{tabular}{ll}
\hline$\tilde{r} / d_{\text {avg }}$ & $\mathcal{N}$ \\
\hline
\end{tabular}

$0.553 \quad 1.47 \times 10^{-3}$

$0.691 \quad 7.57 \times 10^{-4}$

$0.829 \quad 3.46 \times 10^{-4}$

$0.968 \quad 1.34 \times 10^{-4}$

$1.11 \quad 5.16 \times 10^{-5}$

$\begin{array}{ll}1.24 & 2.47 \times 10^{-5}\end{array}$

$1.38 \quad 1.38 \times 10^{-5}$

$1.52 \quad 8.13 \times 10^{-6}$

$1.66 \quad 4.78 \times 10^{-6}$

$1.8 \quad 2.67 \times 10^{-6}$

$1.94 \quad 1.36 \times 10^{-6}$

$2.07 \quad 6.15 \times 10^{-7}$

$2.21 \quad 2.25 \times 10^{-7}$

$2.35 \quad 6.57 \times 10^{-8}$

$2.49 \quad 2.40 \times 10^{-8}$

$2.63 \quad 1.18 \times 10^{-8}$

$2.76 \quad 6.68 \times 10^{-9}$

$2.9 \quad 4.01 \times 10^{-9}$

$3.04 \quad 2.39 \times 10^{-9}$

$3.18+1.33 \times 10^{-9}$

$3.32 \quad 6.03 \times 10^{-10}$

$3.46 \quad 1.80 \times 10^{-10}$

$3.59+5.06 \times 10^{-11}$

$\begin{array}{ll}3.73 & 1.69 \times 10^{-11}\end{array}$

$3.87 \quad 4.61 \times 10^{-12}$

$4.01 \quad 1.13 \times 10^{-12}$

$4.15 \quad 4.72 \times 10^{-13}$

$4.29 \quad 2.56 \times 10^{-13}$

$4.42 \quad 1.52 \times 10^{-13}$

$4.56 \quad 9.09 \times 10^{-14}$

$\begin{array}{ll}4.7 & 5.05 \times 10^{-14}\end{array}$

$4.84 \quad 2.17 \times 10^{-14}$

$4.98+1.39 \times 10^{-16}$

\begin{tabular}{lc}
\hline \hline & $d_{\text {lat }}=11$ \\
\hline$\tilde{r} / d_{\text {avg }}$ & $\mathcal{N}$ \\
\hline 0.106 & $2.35 \times 10^{-2}$ \\
0.211 & $9.16 \times 10^{-3}$ \\
0.317 & $5.47 \times 10^{-3}$ \\
0.423 & $3.40 \times 10^{-3}$ \\
0.528 & $2.11 \times 10^{-3}$ \\
0.634 & $1.27 \times 10^{-3}$ \\
\hline
\end{tabular}

(Table continued) 
TABLE III. (Continued)

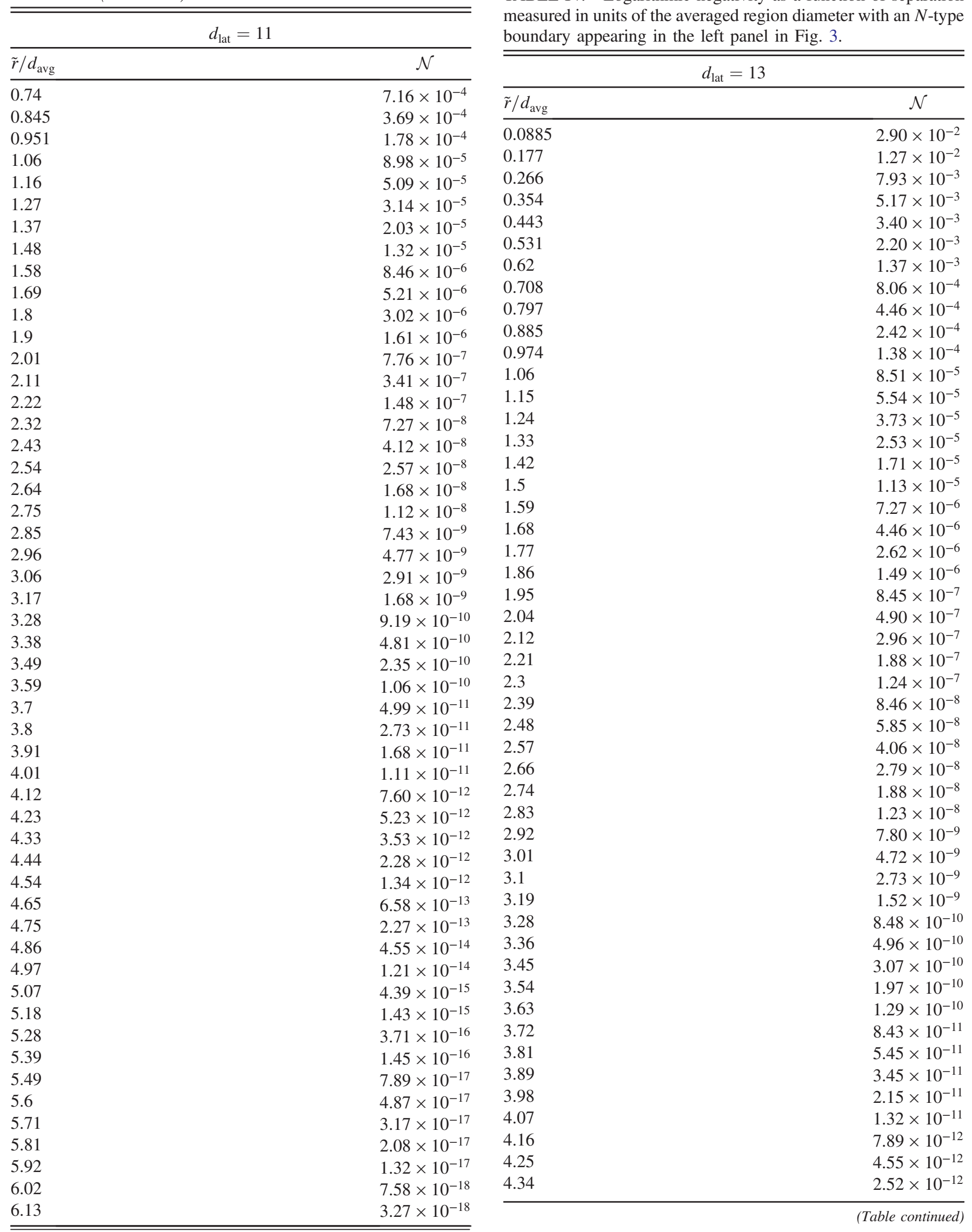


TABLE IV. (Continued)

\begin{tabular}{|c|c|c|c|}
\hline \multicolumn{2}{|c|}{$d_{\text {lat }}=13$} & \multicolumn{2}{|c|}{$d_{\text {lat }}=15$} \\
\hline$\tilde{r} / d_{\mathrm{avg}}$ & $\mathcal{N}$ & $\tilde{r} / d_{\text {avg }}$ & $\mathcal{N}$ \\
\hline 4.43 & $1.36 \times 10^{-12}$ & 1.07 & $8.05 \times 10^{-5}$ \\
\hline 4.51 & $7.28 \times 10^{-13}$ & 1.15 & $5.67 \times 10^{-5}$ \\
\hline 4.6 & $3.76 \times 10^{-13}$ & 1.22 & $4.02 \times 10^{-5}$ \\
\hline 4.69 & $1.85 \times 10^{-13}$ & 1.3 & $2.84 \times 10^{-5}$ \\
\hline 4.78 & $9.15 \times 10^{-14}$ & 1.38 & $1.98 \times 10^{-5}$ \\
\hline 4.87 & $5.04 \times 10^{-14}$ & 1.45 & $1.35 \times 10^{-5}$ \\
\hline 4.96 & $3.11 \times 10^{-14}$ & 1.53 & $8.94 \times 10^{-6}$ \\
\hline 5.05 & $2.08 \times 10^{-14}$ & 1.61 & $5.73 \times 10^{-6}$ \\
\hline 5.13 & $1.45 \times 10^{-14}$ & 1.68 & $3.56 \times 10^{-6}$ \\
\hline 5.22 & $1.04 \times 10^{-14}$ & 1.76 & $2.18 \times 10^{-6}$ \\
\hline 5.31 & $7.45 \times 10^{-15}$ & 1.84 & $1.36 \times 10^{-6}$ \\
\hline 5.4 & $5.30 \times 10^{-15}$ & 1.91 & $8.75 \times 10^{-7}$ \\
\hline 5.49 & $3.67 \times 10^{-15}$ & 1.99 & $5.89 \times 10^{-7}$ \\
\hline 5.58 & $2.41 \times 10^{-15}$ & 2.07 & $4.10 \times 10^{-7}$ \\
\hline 5.67 & $1.46 \times 10^{-15}$ & 2.14 & $2.92 \times 10^{-7}$ \\
\hline 5.75 & $7.89 \times 10^{-16}$ & 2.22 & $2.10 \times 10^{-7}$ \\
\hline 5.84 & $3.85 \times 10^{-16}$ & 2.3 & $1.52 \times 10^{-7}$ \\
\hline 5.93 & $1.71 \times 10^{-16}$ & 2.37 & $1.10 \times 10^{-7}$ \\
\hline 6.02 & $6.10 \times 10^{-17}$ & 2.45 & $7.81 \times 10^{-8}$ \\
\hline 6.11 & $1.64 \times 10^{-17}$ & 2.52 & $5.46 \times 10^{-8}$ \\
\hline 6.2 & $6.17 \times 10^{-18}$ & 2.6 & $3.74 \times 10^{-8}$ \\
\hline 6.28 & $3.19 \times 10^{-18}$ & 2.68 & $2.49 \times 10^{-8}$ \\
\hline 6.37 & $1.87 \times 10^{-18}$ & 2.75 & $1.61 \times 10^{-8}$ \\
\hline 6.46 & $1.13 \times 10^{-18}$ & 2.83 & $1.01 \times 10^{-8}$ \\
\hline 6.55 & $6.74 \times 10^{-19}$ & 2.91 & $6.19 \times 10^{-9}$ \\
\hline 6.64 & $3.68 \times 10^{-19}$ & 2.98 & $3.75 \times 10^{-9}$ \\
\hline 6.73 & $1.70 \times 10^{-19}$ & 3.06 & $2.32 \times 10^{-9}$ \\
\hline 6.82 & $7.10 \times 10^{-20}$ & 3.14 & $1.50 \times 10^{-9}$ \\
\hline 6.9 & $3.36 \times 10^{-20}$ & 3.21 & $1.01 \times 10^{-9}$ \\
\hline 6.99 & $1.81 \times 10^{-20}$ & 3.29 & $6.99 \times 10^{-10}$ \\
\hline 7.08 & $1.02 \times 10^{-20}$ & 3.37 & $4.91 \times 10^{-10}$ \\
\hline 7.17 & $5.45 \times 10^{-21}$ & 3.44 & $3.47 \times 10^{-10}$ \\
\hline 7.26 & $2.35 \times 10^{-21}$ & 3.52 & $2.43 \times 10^{-10}$ \\
\hline 7.35 & $1.61 \times 10^{-22}$ & 3.6 & $1.68 \times 10^{-10}$ \\
\hline \multirow{2}{*}{\multicolumn{2}{|c|}{$d_{\text {lat }}=15$}} & 3.67 & $1.14 \times 10^{-10}$ \\
\hline & & 3.75 & $7.67 \times 10^{-11}$ \\
\hline$\overline{\tilde{r} / d_{\mathrm{avg}}}$ & $\mathcal{N}$ & 3.83 & $5.10 \times 10^{-11}$ \\
\hline 0.0765 & $3.32 \times 10^{-2}$ & $\begin{array}{l}3.9 \\
3.98\end{array}$ & $\begin{array}{l}3.37 \times 10^{-11} \\
2.21 \times 10^{-11}\end{array}$ \\
\hline 0.153 & $1.55 \times 10^{-2}$ & 4.05 & $1.43 \times 10^{-11}$ \\
\hline 0.23 & $1.00 \times 10^{-2}$ & 4.13 & $9.09 \times 10^{-12}$ \\
\hline 0.306 & $6.72 \times 10^{-3}$ & 4.21 & $5.65 \times 10^{-12}$ \\
\hline 0.383 & $4.57 \times 10^{-3}$ & 4.28 & $3.47 \times 10^{-12}$ \\
\hline 0.459 & $3.08 \times 10^{-3}$ & 4.36 & $2.15 \times 10^{-12}$ \\
\hline 0.536 & $2.03 \times 10^{-3}$ & 4.44 & $1.39 \times 10^{-12}$ \\
\hline 0.612 & $1.28 \times 10^{-3}$ & 4.51 & $9.32 \times 10^{-13}$ \\
\hline 0.689 & $7.78 \times 10^{-4}$ & 4.59 & $6.51 \times 10^{-13}$ \\
\hline 0.765 & $4.60 \times 10^{-4}$ & 4.67 & $4.67 \times 10^{-13}$ \\
\hline 0.842 & $2.77 \times 10^{-4}$ & 4.74 & $3.40 \times 10^{-13}$ \\
\hline 0.918 & $1.75 \times 10^{-4}$ & 4.82 & $2.49 \times 10^{-13}$ \\
\hline 0.995 & $1.17 \times 10^{-4}$ & 4.9 & $1.82 \times 10^{-13}$ \\
\hline
\end{tabular}

TABLE IV. (Continued) 
TABLE IV. (Continued)

\begin{tabular}{|c|c|c|c|}
\hline \multicolumn{2}{|c|}{$d_{\text {lat }}=15$} & \multicolumn{2}{|c|}{$\begin{array}{l}\text { measured in units of the averaged region diameter with an } N \text {-type } \\
\text { boundary appearing in the left panel in Fig. } 3 .\end{array}$} \\
\hline$\tilde{r} / d_{\text {avg }}$ & $\mathcal{N}$ & \multicolumn{2}{|c|}{$d_{\text {lat }}=17$} \\
\hline $\begin{array}{l}4.97 \\
5.05\end{array}$ & $\begin{array}{l}1.31 \times 10^{-13} \\
9.22 \times 10^{-14}\end{array}$ & $\tilde{r} / d_{\text {avg }}$ & $\mathcal{N}$ \\
\hline 5.13 & $6.28 \times 10^{-14}$ & 0.0661 & $3.98 \times 10^{-2}$ \\
\hline 5.2 & $4.13 \times 10^{-14}$ & 0.132 & $2.01 \times 10^{-2}$ \\
\hline 5.28 & $2.62 \times 10^{-14}$ & 0.198 & $1.34 \times 10^{-2}$ \\
\hline 5.36 & $1.62 \times 10^{-14}$ & 0.264 & $9.33 \times 10^{-3}$ \\
\hline 5.43 & $9.91 \times 10^{-15}$ & 0.33 & $6.60 \times 10^{-3}$ \\
\hline 5.51 & $5.94 \times 10^{-15}$ & 0.397 & $4.68 \times 10^{-3}$ \\
\hline 5.59 & $3.44 \times 10^{-15}$ & 0.463 & $3.29 \times 10^{-3}$ \\
\hline 5.66 & $1.90 \times 10^{-15}$ & 0.529 & $2.26 \times 10^{-3}$ \\
\hline 5.74 & $1.00 \times 10^{-15}$ & 0.595 & $1.50 \times 10^{-3}$ \\
\hline 5.81 & $5.14 \times 10^{-16}$ & 0.661 & $9.66 \times 10^{-4}$ \\
\hline 5.89 & $2.58 \times 10^{-16}$ & 0.727 & $6.07 \times 10^{-4}$ \\
\hline 5.97 & $1.30 \times 10^{-16}$ & 0.793 & $3.85 \times 10^{-4}$ \\
\hline 6.04 & $6.94 \times 10^{-17}$ & 0.859 & $2.53 \times 10^{-4}$ \\
\hline 6.12 & $4.10 \times 10^{-17}$ & 0.925 & $1.74 \times 10^{-4}$ \\
\hline 6.2 & $2.65 \times 10^{-17}$ & 0.991 & $1.24 \times 10^{-4}$ \\
\hline 6.27 & $1.82 \times 10^{-17}$ & 1.06 & $9.10 \times 10^{-5}$ \\
\hline 6.35 & $1.30 \times 10^{-17}$ & 1.12 & $6.73 \times 10^{-5}$ \\
\hline 6.43 & $9.51 \times 10^{-18}$ & 1.19 & $5.00 \times 10^{-5}$ \\
\hline 6.5 & $6.99 \times 10^{-18}$ & 1.26 & $3.70 \times 10^{-5}$ \\
\hline 6.58 & $5.12 \times 10^{-18}$ & 1.32 & $2.70 \times 10^{-5}$ \\
\hline 6.66 & $3.68 \times 10^{-18}$ & 1.39 & $1.94 \times 10^{-5}$ \\
\hline 6.73 & $2.55 \times 10^{-18}$ & 1.45 & $1.37 \times 10^{-5}$ \\
\hline 6.81 & $1.69 \times 10^{-18}$ & 1.52 & $9.36 \times 10^{-6}$ \\
\hline 6.89 & $1.04 \times 10^{-18}$ & 1.59 & $6.24 \times 10^{-6}$ \\
\hline 6.96 & $5.96 \times 10^{-19}$ & 1.65 & $4.09 \times 10^{-6}$ \\
\hline 7.04 & $3.28 \times 10^{-19}$ & 1.72 & $2.69 \times 10^{-6}$ \\
\hline 7.12 & $1.75 \times 10^{-19}$ & 1.78 & $1.81 \times 10^{-6}$ \\
\hline 7.19 & $8.57 \times 10^{-20}$ & 1.85 & $1.26 \times 10^{-6}$ \\
\hline 7.27 & $3.49 \times 10^{-20}$ & 1.92 & $9.04 \times 10^{-7}$ \\
\hline 7.34 & $1.28 \times 10^{-20}$ & 1.98 & $6.66 \times 10^{-7}$ \\
\hline 7.42 & $6.08 \times 10^{-21}$ & 2.05 & $4.98 \times 10^{-7}$ \\
\hline 7.5 & $3.60 \times 10^{-21}$ & 2.11 & $3.76 \times 10^{-7}$ \\
\hline 7.57 & $2.39 \times 10^{-21}$ & 2.18 & $2.85 \times 10^{-7}$ \\
\hline 7.65 & $1.68 \times 10^{-21}$ & 2.25 & $2.15 \times 10^{-7}$ \\
\hline 7.73 & $1.22 \times 10^{-21}$ & 2.31 & $1.60 \times 10^{-7}$ \\
\hline 7.8 & $8.96 \times 10^{-22}$ & 2.38 & $1.18 \times 10^{-7}$ \\
\hline 7.88 & $6.57 \times 10^{-22}$ & 2.45 & $8.51 \times 10^{-8}$ \\
\hline 7.96 & $4.74 \times 10^{-22}$ & 2.51 & $6.00 \times 10^{-8}$ \\
\hline 8.03 & $3.29 \times 10^{-22}$ & 2.58 & $4.13 \times 10^{-8}$ \\
\hline 8.11 & $2.13 \times 10^{-22}$ & 2.64 & $2.77 \times 10^{-8}$ \\
\hline 8.19 & $1.18 \times 10^{-22}$ & 2.71 & $1.80 \times 10^{-8}$ \\
\hline 8.26 & $4.37 \times 10^{-23}$ & 2.78 & $1.14 \times 10^{-8}$ \\
\hline 8.34 & $8.04 \times 10^{-24}$ & 2.84 & $7.09 \times 10^{-9}$ \\
\hline 8.42 & $2.08 \times 10^{-24}$ & 2.91 & $4.46 \times 10^{-9}$ \\
\hline 8.49 & $4.51 \times 10^{-25}$ & 2.97 & $2.92 \times 10^{-9}$ \\
\hline & & 3.04 & $2.00 \times 10^{-9}$ \\
\hline & & 3.11 & $1.43 \times 10^{-9}$ \\
\hline & & 3.17 & $1.06 \times 10^{-9}$ \\
\hline & & 3.24 & $7.97 \times 10^{-10}$ \\
\hline
\end{tabular}

TABLE V. Logarithmic negativity as a function of separation measured in units of the averaged region diameter with an $N$-type boundary appearing in the left panel in Fig. 3. 
TABLE V. (Continued)

\begin{tabular}{|c|c|c|c|}
\hline \multicolumn{2}{|c|}{$d_{\text {lat }}=17$} & \multicolumn{2}{|c|}{$d_{\text {lat }}=17$} \\
\hline$\tilde{r} / d_{\text {avg }}$ & $\mathcal{N}$ & $\tilde{r} / d_{\text {avg }}$ & $\mathcal{N}$ \\
\hline 3.3 & $6.07 \times 10^{-10}$ & 6.68 & $5.28 \times 10^{-18}$ \\
\hline 3.37 & $4.65 \times 10^{-10}$ & 6.74 & $3.32 \times 10^{-18}$ \\
\hline 3.44 & $3.55 \times 10^{-10}$ & 6.81 & $2.08 \times 10^{-18}$ \\
\hline 3.5 & $2.69 \times 10^{-10}$ & 6.87 & $1.30 \times 10^{-18}$ \\
\hline 3.57 & $2.01 \times 10^{-10}$ & 6.94 & $8.19 \times 10^{-19}$ \\
\hline 3.63 & $1.47 \times 10^{-10}$ & 7.01 & $5.17 \times 10^{-19}$ \\
\hline 3.7 & $1.05 \times 10^{-10}$ & 7.07 & $3.26 \times 10^{-19}$ \\
\hline 3.77 & $7.28 \times 10^{-11}$ & 7.14 & $2.04 \times 10^{-19}$ \\
\hline 3.83 & $4.92 \times 10^{-11}$ & 7.2 & $1.25 \times 10^{-19}$ \\
\hline 3.9 & $3.25 \times 10^{-11}$ & 7.27 & $7.43 \times 10^{-20}$ \\
\hline 3.97 & $2.10 \times 10^{-11}$ & 7.34 & $4.37 \times 10^{-20}$ \\
\hline 4.03 & $1.35 \times 10^{-11}$ & 7.4 & $2.60 \times 10^{-20}$ \\
\hline 4.1 & $8.66 \times 10^{-12}$ & 7.47 & $1.61 \times 10^{-20}$ \\
\hline 4.16 & $5.61 \times 10^{-12}$ & 7.53 & $1.04 \times 10^{-20}$ \\
\hline 4.23 & $3.70 \times 10^{-12}$ & 7.6 & $7.05 \times 10^{-21}$ \\
\hline 4.3 & $2.50 \times 10^{-12}$ & 7.67 & $4.88 \times 10^{-21}$ \\
\hline 4.36 & $1.73 \times 10^{-12}$ & 7.73 & $3.41 \times 10^{-21}$ \\
\hline 4.43 & $1.24 \times 10^{-12}$ & 7.8 & $2.39 \times 10^{-21}$ \\
\hline 4.49 & $9.05 \times 10^{-13}$ & 7.86 & $1.65 \times 10^{-21}$ \\
\hline 4.56 & $6.75 \times 10^{-13}$ & 7.93 & $1.12 \times 10^{-21}$ \\
\hline 4.63 & $5.10 \times 10^{-13}$ & 8. & $7.44 \times 10^{-22}$ \\
\hline 4.69 & $3.89 \times 10^{-13}$ & 8.06 & $4.79 \times 10^{-22}$ \\
\hline 4.76 & $2.96 \times 10^{-13}$ & 8.13 & $3.00 \times 10^{-22}$ \\
\hline 4.82 & $2.24 \times 10^{-13}$ & 8.2 & $1.84 \times 10^{-22}$ \\
\hline 4.89 & $1.68 \times 10^{-13}$ & 8.26 & $1.08 \times 10^{-22}$ \\
\hline 4.96 & $1.24 \times 10^{-13}$ & 8.33 & $5.97 \times 10^{-23}$ \\
\hline 5.02 & $8.98 \times 10^{-14}$ & 8.39 & $2.91 \times 10^{-23}$ \\
\hline 5.09 & $6.33 \times 10^{-14}$ & 8.46 & $1.25 \times 10^{-23}$ \\
\hline 5.16 & $4.37 \times 10^{-14}$ & 8.53 & $5.85 \times 10^{-24}$ \\
\hline 5.22 & $2.94 \times 10^{-14}$ & 8.59 & $3.31 \times 10^{-24}$ \\
\hline 5.29 & $1.94 \times 10^{-14}$ & 8.66 & $2.14 \times 10^{-24}$ \\
\hline 5.35 & $1.25 \times 10^{-14}$ & 8.72 & $1.49 \times 10^{-24}$ \\
\hline 5.42 & $7.81 \times 10^{-15}$ & 8.79 & $1.08 \times 10^{-24}$ \\
\hline 5.49 & $4.77 \times 10^{-15}$ & 8.86 & $7.99 \times 10^{-25}$ \\
\hline 5.55 & $2.92 \times 10^{-15}$ & 8.92 & $5.96 \times 10^{-25}$ \\
\hline 5.62 & $1.86 \times 10^{-15}$ & 8.99 & $4.44 \times 10^{-25}$ \\
\hline 5.68 & $1.24 \times 10^{-15}$ & 9.05 & $3.25 \times 10^{-25}$ \\
\hline 5.75 & $8.70 \times 10^{-16}$ & 9.12 & $2.30 \times 10^{-25}$ \\
\hline 5.82 & $6.31 \times 10^{-16}$ & 9.19 & $1.54 \times 10^{-25}$ \\
\hline 5.88 & $4.68 \times 10^{-16}$ & 9.25 & $9.20 \times 10^{-26}$ \\
\hline 5.95 & $3.51 \times 10^{-16}$ & 9.32 & $4.39 \times 10^{-26}$ \\
\hline 6.01 & $2.65 \times 10^{-16}$ & 9.38 & $1.04 \times 10^{-26}$ \\
\hline 6.08 & $1.99 \times 10^{-16}$ & 9.45 & $1.06 \times 10^{-27}$ \\
\hline 6.15 & $1.47 \times 10^{-16}$ & 9.52 & $3.19 \times 10^{-28}$ \\
\hline 6.21 & $1.07 \times 10^{-16}$ & 9.58 & $1.11 \times 10^{-28}$ \\
\hline 6.28 & $7.54 \times 10^{-17}$ & 9.65 & $1.39 \times 10^{-29}$ \\
\hline 6.34 & $5.12 \times 10^{-17}$ & & \\
\hline 6.41 & $3.35 \times 10^{-17}$ & & \\
\hline 6.48 & $2.13 \times 10^{-17}$ & & \\
\hline 6.54 & $1.34 \times 10^{-17}$ & & \\
\hline 6.61 & $8.39 \times 10^{-18}$ & & \\
\hline
\end{tabular}

TABLE V. (Continued) 
TABLE VI. Logarithmic negativity as a function of separation measured in units of the averaged region diameter with an $N$-type boundary appearing in the left panel in Fig. 3 .

\begin{tabular}{|c|c|}
\hline \multicolumn{2}{|c|}{$d_{\text {lat }}=19$} \\
\hline$\tilde{r} / d_{\text {avg }}$ & $\mathcal{N}$ \\
\hline 0.058 & $4.98 \times 10^{-2}$ \\
\hline 0.116 & $2.61 \times 10^{-2}$ \\
\hline 0.174 & $1.75 \times 10^{-2}$ \\
\hline 0.232 & $1.24 \times 10^{-2}$ \\
\hline 0.29 & $8.94 \times 10^{-3}$ \\
\hline 0.348 & $6.52 \times 10^{-3}$ \\
\hline 0.406 & $4.75 \times 10^{-3}$ \\
\hline 0.464 & $3.43 \times 10^{-3}$ \\
\hline 0.522 & $2.43 \times 10^{-3}$ \\
\hline 0.58 & $1.69 \times 10^{-3}$ \\
\hline 0.638 & $1.15 \times 10^{-3}$ \\
\hline 0.697 & $7.75 \times 10^{-4}$ \\
\hline 0.755 & $5.28 \times 10^{-4}$ \\
\hline 0.813 & $3.69 \times 10^{-4}$ \\
\hline 0.871 & $2.64 \times 10^{-4}$ \\
\hline 0.929 & $1.94 \times 10^{-4}$ \\
\hline 0.987 & $1.45 \times 10^{-4}$ \\
\hline 1.04 & $1.10 \times 10^{-4}$ \\
\hline 1.1 & $8.36 \times 10^{-5}$ \\
\hline 1.16 & $6.36 \times 10^{-5}$ \\
\hline 1.22 & $4.82 \times 10^{-5}$ \\
\hline 1.28 & $3.61 \times 10^{-5}$ \\
\hline 1.34 & $2.68 \times 10^{-5}$ \\
\hline 1.39 & $1.95 \times 10^{-5}$ \\
\hline 1.45 & $1.40 \times 10^{-5}$ \\
\hline 1.51 & $9.92 \times 10^{-6}$ \\
\hline 1.57 & $6.94 \times 10^{-6}$ \\
\hline 1.63 & $4.86 \times 10^{-6}$ \\
\hline 1.68 & $3.43 \times 10^{-6}$ \\
\hline 1.74 & $2.47 \times 10^{-6}$ \\
\hline 1.8 & $1.81 \times 10^{-6}$ \\
\hline 1.86 & $1.35 \times 10^{-6}$ \\
\hline 1.92 & $1.02 \times 10^{-6}$ \\
\hline 1.97 & $7.76 \times 10^{-7}$ \\
\hline 2.03 & $5.95 \times 10^{-7}$ \\
\hline 2.09 & $4.57 \times 10^{-7}$ \\
\hline 2.15 & $3.50 \times 10^{-7}$ \\
\hline 2.21 & $2.67 \times 10^{-7}$ \\
\hline 2.26 & $2.02 \times 10^{-7}$ \\
\hline 2.32 & $1.51 \times 10^{-7}$ \\
\hline 2.38 & $1.12 \times 10^{-7}$ \\
\hline 2.44 & $8.15 \times 10^{-8}$ \\
\hline 2.5 & $5.84 \times 10^{-8}$ \\
\hline 2.55 & $4.13 \times 10^{-8}$ \\
\hline 2.61 & $2.89 \times 10^{-8}$ \\
\hline 2.67 & $2.02 \times 10^{-8}$ \\
\hline 2.73 & $1.42 \times 10^{-8}$ \\
\hline 2.79 & $1.02 \times 10^{-8}$ \\
\hline 2.84 & $7.45 \times 10^{-9}$ \\
\hline
\end{tabular}

TABLE VI. (Continued)

\begin{tabular}{|c|c|}
\hline \multicolumn{2}{|c|}{$d_{\text {lat }}=19$} \\
\hline$\tilde{r} / d_{\mathrm{avg}}$ & $\mathcal{N}$ \\
\hline 2.9 & $5.54 \times 10^{-9}$ \\
\hline 2.96 & $4.18 \times 10^{-9}$ \\
\hline 3.02 & $3.19 \times 10^{-9}$ \\
\hline 3.08 & $2.45 \times 10^{-9}$ \\
\hline 3.13 & $1.89 \times 10^{-9}$ \\
\hline 3.19 & $1.46 \times 10^{-9}$ \\
\hline 3.25 & $1.12 \times 10^{-9}$ \\
\hline 3.31 & $8.56 \times 10^{-10}$ \\
\hline 3.37 & $6.51 \times 10^{-10}$ \\
\hline 3.42 & $4.90 \times 10^{-10}$ \\
\hline 3.48 & $3.66 \times 10^{-10}$ \\
\hline 3.54 & $2.70 \times 10^{-10}$ \\
\hline 3.6 & $1.96 \times 10^{-10}$ \\
\hline 3.66 & $1.41 \times 10^{-10}$ \\
\hline 3.71 & $9.99 \times 10^{-11}$ \\
\hline 3.77 & $6.99 \times 10^{-11}$ \\
\hline 3.83 & $4.85 \times 10^{-11}$ \\
\hline 3.89 & $3.37 \times 10^{-11}$ \\
\hline 3.95 & $2.37 \times 10^{-11}$ \\
\hline 4.01 & $1.69 \times 10^{-11}$ \\
\hline 4.06 & $1.23 \times 10^{-11}$ \\
\hline 4.12 & $9.14 \times 10^{-12}$ \\
\hline 4.18 & $6.88 \times 10^{-12}$ \\
\hline 4.24 & $5.23 \times 10^{-12}$ \\
\hline 4.3 & $3.99 \times 10^{-12}$ \\
\hline 4.35 & $3.05 \times 10^{-12}$ \\
\hline 4.41 & $2.31 \times 10^{-12}$ \\
\hline 4.47 & $1.74 \times 10^{-12}$ \\
\hline 4.53 & $1.30 \times 10^{-12}$ \\
\hline 4.59 & $9.59 \times 10^{-13}$ \\
\hline 4.64 & $7.02 \times 10^{-13}$ \\
\hline 4.7 & $5.12 \times 10^{-13}$ \\
\hline 4.76 & $3.73 \times 10^{-13}$ \\
\hline 4.82 & $2.73 \times 10^{-13}$ \\
\hline 4.88 & $1.99 \times 10^{-13}$ \\
\hline 4.93 & $1.46 \times 10^{-13}$ \\
\hline 4.99 & $1.06 \times 10^{-13}$ \\
\hline 5.05 & $7.65 \times 10^{-14}$ \\
\hline 5.11 & $5.46 \times 10^{-14}$ \\
\hline 5.17 & $3.87 \times 10^{-14}$ \\
\hline 5.22 & $2.74 \times 10^{-14}$ \\
\hline 5.28 & $1.94 \times 10^{-14}$ \\
\hline 5.34 & $1.39 \times 10^{-14}$ \\
\hline 5.4 & $1.02 \times 10^{-14}$ \\
\hline 5.46 & $7.53 \times 10^{-15}$ \\
\hline 5.51 & $5.67 \times 10^{-15}$ \\
\hline 5.57 & $4.32 \times 10^{-15}$ \\
\hline 5.63 & $3.32 \times 10^{-15}$ \\
\hline 5.69 & $2.56 \times 10^{-15}$ \\
\hline 5.75 & $1.97 \times 10^{-15}$ \\
\hline 5.8 & $1.52 \times 10^{-15}$ \\
\hline
\end{tabular}


TABLE VI. (Continued)

\begin{tabular}{|c|c|}
\hline \multicolumn{2}{|c|}{$d_{\text {lat }}=19$} \\
\hline$\tilde{r} / d_{\mathrm{avg}}$ & $\mathcal{N}$ \\
\hline 5.86 & $1.16 \times 10^{-15}$ \\
\hline 5.92 & $8.77 \times 10^{-16}$ \\
\hline 5.98 & $6.57 \times 10^{-16}$ \\
\hline 6.04 & $4.86 \times 10^{-16}$ \\
\hline 6.09 & $3.56 \times 10^{-16}$ \\
\hline 6.15 & $2.58 \times 10^{-16}$ \\
\hline 6.21 & $1.85 \times 10^{-16}$ \\
\hline 6.27 & $1.32 \times 10^{-16}$ \\
\hline 6.33 & $9.31 \times 10^{-17}$ \\
\hline 6.38 & $6.51 \times 10^{-17}$ \\
\hline 6.44 & $4.50 \times 10^{-17}$ \\
\hline 6.5 & $3.08 \times 10^{-17}$ \\
\hline 6.56 & $2.08 \times 10^{-17}$ \\
\hline 6.62 & $1.40 \times 10^{-17}$ \\
\hline 6.68 & $9.37 \times 10^{-18}$ \\
\hline 6.73 & $6.28 \times 10^{-18}$ \\
\hline 6.79 & $4.23 \times 10^{-18}$ \\
\hline 6.85 & $2.85 \times 10^{-18}$ \\
\hline 6.91 & $1.94 \times 10^{-18}$ \\
\hline 6.97 & $1.32 \times 10^{-18}$ \\
\hline 7.02 & $9.18 \times 10^{-19}$ \\
\hline 7.08 & $6.47 \times 10^{-19}$ \\
\hline 7.14 & $4.65 \times 10^{-19}$ \\
\hline 7.2 & $3.41 \times 10^{-19}$ \\
\hline 7.26 & $2.54 \times 10^{-19}$ \\
\hline 7.31 & $1.91 \times 10^{-19}$ \\
\hline 7.37 & $1.46 \times 10^{-19}$ \\
\hline 7.43 & $1.11 \times 10^{-19}$ \\
\hline 7.49 & $8.53 \times 10^{-20}$ \\
\hline 7.55 & $6.50 \times 10^{-20}$ \\
\hline 7.6 & $4.91 \times 10^{-20}$ \\
\hline 7.66 & $3.66 \times 10^{-20}$ \\
\hline 7.72 & $2.67 \times 10^{-20}$ \\
\hline 7.78 & $1.89 \times 10^{-20}$ \\
\hline 7.84 & $1.30 \times 10^{-20}$ \\
\hline 7.89 & $8.61 \times 10^{-21}$ \\
\hline 7.95 & $5.52 \times 10^{-21}$ \\
\hline 8.01 & $3.44 \times 10^{-21}$ \\
\hline 8.07 & $2.10 \times 10^{-21}$ \\
\hline 8.13 & $1.27 \times 10^{-21}$ \\
\hline 8.18 & $7.92 \times 10^{-22}$ \\
\hline 8.24 & $5.16 \times 10^{-22}$ \\
\hline 8.3 & $3.53 \times 10^{-22}$ \\
\hline 8.36 & $2.50 \times 10^{-22}$ \\
\hline 8.42 & $1.81 \times 10^{-22}$ \\
\hline 8.47 & $1.33 \times 10^{-22}$ \\
\hline 8.53 & $9.83 \times 10^{-23}$ \\
\hline 8.59 & $7.24 \times 10^{-23}$ \\
\hline 8.65 & $5.28 \times 10^{-23}$ \\
\hline 8.71 & $3.79 \times 10^{-23}$ \\
\hline 8.76 & $2.65 \times 10^{-23}$ \\
\hline
\end{tabular}

TABLE VI. (Continued)

\begin{tabular}{lc}
\hline \hline & $d_{\text {lat }}=19$ \\
\hline$\tilde{r} / d_{\text {avg }}$ & $\mathcal{N}$ \\
\hline 8.82 & $1.80 \times 10^{-23}$ \\
8.88 & $1.17 \times 10^{-23}$ \\
8.94 & $7.27 \times 10^{-24}$ \\
\hline \hline
\end{tabular}

TABLE VII. Logarithmic negativity as a function of separation measured in units of the averaged region diameter with an $N$-type boundary appearing in the left panel in Fig. 3.

\begin{tabular}{|c|c|}
\hline \multicolumn{2}{|c|}{$d_{\text {lat }}=21$} \\
\hline$\tilde{r} / d_{\text {avg }}$ & $\mathcal{N}$ \\
\hline 2.68 & $1.72 \times 10^{-8}$ \\
\hline 2.99 & $3.64 \times 10^{-9}$ \\
\hline 3.3 & $8.75 \times 10^{-10}$ \\
\hline 3.61 & $1.52 \times 10^{-10}$ \\
\hline 3.92 & $2.60 \times 10^{-11}$ \\
\hline 4.23 & $6.18 \times 10^{-12}$ \\
\hline 4.54 & $1.33 \times 10^{-12}$ \\
\hline 4.85 & $2.17 \times 10^{-13}$ \\
\hline 5.16 & $3.24 \times 10^{-14}$ \\
\hline 5.47 & $6.89 \times 10^{-15}$ \\
\hline 5.78 & $1.50 \times 10^{-15}$ \\
\hline 6.09 & $2.66 \times 10^{-16}$ \\
\hline 6.4 & $3.76 \times 10^{-17}$ \\
\hline 6.71 & $6.37 \times 10^{-18}$ \\
\hline 7.02 & $1.56 \times 10^{-18}$ \\
\hline 7.33 & $3.22 \times 10^{-19}$ \\
\hline 7.64 & $4.47 \times 10^{-20}$ \\
\hline 7.95 & $6.12 \times 10^{-21}$ \\
\hline 8.26 & $9.22 \times 10^{-22}$ \\
\hline 8.57 & $1.34 \times 10^{-22}$ \\
\hline \multicolumn{2}{|c|}{$d_{\text {lat }}=23$} \\
\hline$\tilde{r} / d_{\mathrm{avg}}$ & $\mathcal{N}$ \\
\hline 2.69 & $1.58 \times 10^{-8}$ \\
\hline 2.97 & $4.29 \times 10^{-9}$ \\
\hline 3.26 & $1.12 \times 10^{-9}$ \\
\hline 3.54 & $2.21 \times 10^{-10}$ \\
\hline 3.82 & $4.56 \times 10^{-11}$ \\
\hline 4.11 & $1.18 \times 10^{-11}$ \\
\hline 4.39 & $2.77 \times 10^{-12}$ \\
\hline 4.67 & $5.72 \times 10^{-13}$ \\
\hline 4.96 & $1.12 \times 10^{-13}$ \\
\hline
\end{tabular}


TABLE VII. (Continued)

\begin{tabular}{|c|c|c|c|}
\hline \multicolumn{2}{|c|}{$d_{\text {lat }}=23$} & \multicolumn{2}{|c|}{$d_{\text {lat }}=27$} \\
\hline$\tilde{r} / d_{\mathrm{avg}}$ & $\mathcal{N}$ & $\tilde{r} / d_{\text {avg }}$ & $\mathcal{N}$ \\
\hline 5.24 & $2.91 \times 10^{-14}$ & 4.08 & $1.39 \times 10^{-11}$ \\
\hline 5.52 & $7.52 \times 10^{-15}$ & 4.32 & $3.81 \times 10^{-12}$ \\
\hline 5.81 & $1.44 \times 10^{-15}$ & 4.56 & $9.90 \times 10^{-13}$ \\
\hline 6.09 & $2.53 \times 10^{-16}$ & 4.8 & $2.92 \times 10^{-13}$ \\
\hline 6.37 & $5.22 \times 10^{-17}$ & 5.04 & $9.12 \times 10^{-14}$ \\
\hline 6.66 & $1.37 \times 10^{-17}$ & 5.27 & $2.64 \times 10^{-14}$ \\
\hline 6.94 & $2.87 \times 10^{-18}$ & 5.51 & $7.18 \times 10^{-15}$ \\
\hline 7.22 & $4.72 \times 10^{-19}$ & 5.75 & $1.89 \times 10^{-15}$ \\
\hline 7.51 & $8.00 \times 10^{-20}$ & 5.99 & $5.22 \times 10^{-16}$ \\
\hline 7.79 & $1.60 \times 10^{-20}$ & 6.23 & $1.51 \times 10^{-16}$ \\
\hline 8.07 & $3.96 \times 10^{-21}$ & 6.46 & $3.98 \times 10^{-17}$ \\
\hline 8.35 & $9.64 \times 10^{-22}$ & 6.7 & $1.09 \times 10^{-17}$ \\
\hline 8.64 & $1.70 \times 10^{-22}$ & 6.94 & $3.02 \times 10^{-18}$ \\
\hline & & 7.18 & $7.98 \times 10^{-19}$ \\
\hline \multicolumn{2}{|c|}{$d_{\text {lat }}=25$} & 7.41 & $2.28 \times 10^{-19}$ \\
\hline$\tilde{r} / d_{\text {avg }}$ & $\mathcal{N}$ & $\begin{array}{l}7.65 \\
7.89\end{array}$ & $\begin{array}{l}7.19 \times 10^{-20} \\
20 \times 10^{-20}\end{array}$ \\
\hline 2.7 & $1.53 \times 10^{-8}$ & 8.13 & $\begin{array}{l}2.20 \times 10^{-20} \\
5.56 \times 10^{-21}\end{array}$ \\
\hline 2.96 & $4.59 \times 10^{-9}$ & 8.37 & $1.28 \times 10^{-21}$ \\
\hline 3.22 & $1.20 \times 10^{-9}$ & & \\
\hline 3.48 & $2.61 \times 10^{-10}$ & \multicolumn{2}{|c|}{$d_{\text {lat }}=29$} \\
\hline 3.74 & $6.64 \times 10^{-11}$ & $\overline{\tilde{r} / d_{\mathrm{avg}}}$ & $\mathcal{N}$ \\
\hline 4.01 & & $\frac{1}{2} u_{\text {avg }}$ & $\frac{0}{20}$ \\
\hline 4.27 & $5.25 \times 10^{-12}$ & 2.65 & $2.30 \times 10^{-8}$ \\
\hline 4.53 & $1.19 \times 10^{-12}$ & 2.87 & $8.13 \times 10^{-9}$ \\
\hline 4.79 & $2.65 \times 10^{-13}$ & 3.09 & $2.53 \times 10^{-9}$ \\
\hline 5.05 & $7.62 \times 10^{-14}$ & 3.31 & $6.95 \times 10^{-10}$ \\
\hline 5.31 & $2.18 \times 10^{-14}$ & 3.53 & $2.14 \times 10^{-10}$ \\
\hline 5.57 & $5.10 \times 10^{-15}$ & 3.75 & $7.51 \times 10^{-11}$ \\
\hline 5.84 & $1.15 \times 10^{-15}$ & 3.97 & $2.55 \times 10^{-11}$ \\
\hline 6.1 & $2.63 \times 10^{-16}$ & 4.19 & $7.75 \times 10^{-12}$ \\
\hline 6.36 & $6.85 \times 10^{-17}$ & 4.41 & $2.2 \times 10^{-12}$ \\
\hline 6.62 & $1.89 \times 10^{-17}$ & 4.63 & $6.91 \times 10^{-13}$ \\
\hline 6.88 & $4.86 \times 10^{-18}$ & 4.85 & $2.35 \times 10^{-13}$ \\
\hline 7.14 & $1.10 \times 10^{-18}$ & 5.07 & $7.55 \times 10^{-14}$ \\
\hline 7.4 & $2.44 \times 10^{-19}$ & 5.29 & $2.27 \times 10^{-14}$ \\
\hline 7.66 & $5.84 \times 10^{-20}$ & 5.51 & $6.62 \times 10^{-15}$ \\
\hline 7.93 & $1.46 \times 10^{-20}$ & 5.74 & $2 . \times 10^{-15}$ \\
\hline 8.19 & $3.36 \times 10^{-21}$ & 5.96 & $6.81 \times 10^{-16}$ \\
\hline 8.45 & $7.29 \times 10^{-22}$ & 6.18 & $2.29 \times 10^{-16}$ \\
\hline \multirow[t]{3}{*}{8.71} & $1.53 \times 10^{-22}$ & 6.4 & $6.81 \times 10^{-17}$ \\
\hline & & 6.62 & $1.99 \times 10^{-17}$ \\
\hline & $d_{\text {lat }}=27$ & 6.84 & $5.95 \times 10^{-18}$ \\
\hline$\tilde{r} / d_{\text {avg }}$ & $\mathcal{N}$ & $\begin{array}{l}7.06 \\
728\end{array}$ & $\begin{array}{l}1.79 \times 10^{-18} \\
568 \times 10^{-19}\end{array}$ \\
\hline 2.66 & $2.07 \times 10^{-8}$ & $\begin{array}{l}1.28 \\
7.5\end{array}$ & $\begin{array}{l}5.68 \times 10^{-19} \\
1.82 \times 10^{-19}\end{array}$ \\
\hline 2.89 & $6.73 \times 10^{-9}$ & 7.72 & $5.8 \times 10^{-20}$ \\
\hline 3.13 & $1.98 \times 10^{-9}$ & 7.94 & $1.87 \times 10^{-20}$ \\
\hline 3.37 & $5.11 \times 10^{-10}$ & 8.16 & $5.68 \times 10^{-21}$ \\
\hline 3.61 & $1.39 \times 10^{-10}$ & 8.38 & $1.55 \times 10^{-21}$ \\
\hline 3.85 & $4.44 \times 10^{-11}$ & & \\
\hline
\end{tabular}

TABLE VII. (Continued)

(Table continued) 
TABLE VIII. Logarithmic negativity as a function of separation measured in units of the averaged region diameter with an $N$-type boundary appearing in the left panel in Fig. 3.

\begin{tabular}{|c|c|}
\hline \multicolumn{2}{|c|}{$d_{\text {lat }}=31$} \\
\hline$\tilde{r} / d_{\text {avg }}$ & $\mathcal{N}$ \\
\hline 2.63 & $2.66 \times 10^{-8}$ \\
\hline 2.8 & $1.19 \times 10^{-8}$ \\
\hline 2.97 & $4.92 \times 10^{-9}$ \\
\hline 3.14 & $1.82 \times 10^{-9}$ \\
\hline 3.31 & $6.67 \times 10^{-10}$ \\
\hline 3.48 & $2.73 \times 10^{-10}$ \\
\hline 3.65 & $1.22 \times 10^{-10}$ \\
\hline 3.82 & $5.47 \times 10^{-11}$ \\
\hline 3.99 & $2.28 \times 10^{-11}$ \\
\hline 4.16 & $8.64 \times 10^{-12}$ \\
\hline 4.33 & $3.22 \times 10^{-12}$ \\
\hline 4.5 & $1.33 \times 10^{-12}$ \\
\hline 4.67 & $5.97 \times 10^{-13}$ \\
\hline 4.84 & $2.64 \times 10^{-13}$ \\
\hline 5.01 & $1.08 \times 10^{-13}$ \\
\hline 5.18 & $4.11 \times 10^{-14}$ \\
\hline 5.36 & $1.49 \times 10^{-14}$ \\
\hline 5.53 & $5.50 \times 10^{-15}$ \\
\hline 5.7 & $2.29 \times 10^{-15}$ \\
\hline 5.87 & $1.02 \times 10^{-15}$ \\
\hline 6.04 & $4.45 \times 10^{-16}$ \\
\hline 6.21 & $1.77 \times 10^{-16}$ \\
\hline 6.38 & $6.85 \times 10^{-17}$ \\
\hline 6.55 & $2.70 \times 10^{-17}$ \\
\hline 6.72 & $1.08 \times 10^{-17}$ \\
\hline 6.89 & $4.44 \times 10^{-18}$ \\
\hline 7.06 & $1.89 \times 10^{-18}$ \\
\hline 7.23 & $7.85 \times 10^{-19}$ \\
\hline 7.4 & $2.98 \times 10^{-19}$ \\
\hline 7.57 & $1.06 \times 10^{-19}$ \\
\hline 7.74 & $3.91 \times 10^{-20}$ \\
\hline 7.91 & $1.51 \times 10^{-20}$ \\
\hline 8.08 & $6.02 \times 10^{-21}$ \\
\hline 8.25 & $2.53 \times 10^{-21}$ \\
\hline 8.43 & $1.10 \times 10^{-21}$ \\
\hline \multicolumn{2}{|c|}{$d_{\text {lat }}=33$} \\
\hline$\tilde{r} / d_{\text {avg }}$ & $\mathcal{N}$ \\
\hline 2.64 & $2.55 \times 10^{-8}$ \\
\hline 2.8 & $1.15 \times 10^{-8}$ \\
\hline 2.96 & $4.78 \times 10^{-9}$ \\
\hline 3.12 & $1.85 \times 10^{-9}$ \\
\hline 3.28 & $7.49 \times 10^{-10}$ \\
\hline 3.44 & $3.36 \times 10^{-10}$ \\
\hline 3.6 & $1.58 \times 10^{-10}$ \\
\hline 3.76 & $7.28 \times 10^{-11}$ \\
\hline 3.92 & $3.10 \times 10^{-11}$ \\
\hline 4.08 & $1.23 \times 10^{-11}$ \\
\hline
\end{tabular}

TABLE VIII. (Continued)

\begin{tabular}{|c|c|}
\hline \multicolumn{2}{|c|}{$d_{\text {lat }}=33$} \\
\hline$\tilde{r} / d_{\text {avg }}$ & $\mathcal{N}$ \\
\hline 4.24 & $4.93 \times 10^{-12}$ \\
\hline 4.4 & $2.17 \times 10^{-12}$ \\
\hline 4.56 & $1.02 \times 10^{-12}$ \\
\hline 4.72 & $4.77 \times 10^{-13}$ \\
\hline 4.88 & $2.10 \times 10^{-13}$ \\
\hline 5.05 & $8.69 \times 10^{-14}$ \\
\hline 5.21 & $3.42 \times 10^{-14}$ \\
\hline 5.37 & $1.34 \times 10^{-14}$ \\
\hline 5.53 & $5.67 \times 10^{-15}$ \\
\hline 5.69 & $2.62 \times 10^{-15}$ \\
\hline 5.85 & $1.22 \times 10^{-15}$ \\
\hline 6.01 & $5.39 \times 10^{-16}$ \\
\hline 6.17 & $2.25 \times 10^{-16}$ \\
\hline 6.33 & $9.31 \times 10^{-17}$ \\
\hline 6.49 & $3.91 \times 10^{-17}$ \\
\hline 6.65 & $1.67 \times 10^{-17}$ \\
\hline 6.81 & $7.22 \times 10^{-18}$ \\
\hline 6.97 & $3.12 \times 10^{-18}$ \\
\hline 7.13 & $1.32 \times 10^{-18}$ \\
\hline 7.29 & $5.53 \times 10^{-19}$ \\
\hline 7.46 & $2.27 \times 10^{-19}$ \\
\hline 7.62 & $9.33 \times 10^{-20}$ \\
\hline 7.78 & $3.93 \times 10^{-20}$ \\
\hline 7.94 & $1.69 \times 10^{-20}$ \\
\hline 8.1 & $7.37 \times 10^{-21}$ \\
\hline 8.26 & $3.14 \times 10^{-21}$ \\
\hline 8.42 & $1.28 \times 10^{-21}$ \\
\hline \multicolumn{2}{|c|}{$d_{\text {lat }}=35$} \\
\hline$\overline{\tilde{r} / d_{\mathrm{avg}}}$ & $\mathcal{N}$ \\
\hline 2.63 & $2.64 \times 10^{-8}$ \\
\hline 2.81 & $1.06 \times 10^{-8}$ \\
\hline 2.99 & $3.85 \times 10^{-9}$ \\
\hline 3.17 & $1.36 \times 10^{-9}$ \\
\hline 3.35 & $5.30 \times 10^{-10}$ \\
\hline 3.53 & $2.21 \times 10^{-10}$ \\
\hline 3.71 & $9.00 \times 10^{-11}$ \\
\hline 3.89 & $3.41 \times 10^{-11}$ \\
\hline 4.07 & $1.21 \times 10^{-11}$ \\
\hline 4.26 & $4.44 \times 10^{-12}$ \\
\hline 4.44 & $1.81 \times 10^{-12}$ \\
\hline 4.62 & $7.51 \times 10^{-13}$ \\
\hline 4.8 & $2.98 \times 10^{-13}$ \\
\hline 4.98 & $1.13 \times 10^{-13}$ \\
\hline 5.16 & $4.10 \times 10^{-14}$ \\
\hline 5.34 & $1.53 \times 10^{-14}$ \\
\hline 5.52 & $6.01 \times 10^{-15}$ \\
\hline 5.7 & $2.41 \times 10^{-15}$ \\
\hline 5.89 & $9.52 \times 10^{-16}$ \\
\hline 6.07 & $3.73 \times 10^{-16}$ \\
\hline
\end{tabular}


TABLE VIII. (Continued)

\begin{tabular}{|c|c|c|c|}
\hline \multicolumn{2}{|c|}{$d_{\text {lat }}=35$} & \multicolumn{2}{|c|}{$d_{\text {lat }}=37$} \\
\hline$\tilde{r} / d_{\text {avg }}$ & $\mathcal{N}$ & $\tilde{r} / d_{\text {avg }}$ & $\mathcal{N}$ \\
\hline 6.25 & $1.41 \times 10^{-16}$ & 8.26 & $3.84 \times 10^{-21}$ \\
\hline 6.43 & $5.17 \times 10^{-17}$ & 8.43 & $1.57 \times 10^{-21}$ \\
\hline 6.61 & $1.97 \times 10^{-17}$ & & \\
\hline 6.79 & $7.85 \times 10^{-18}$ & \multicolumn{2}{|c|}{$d_{\text {lat }}=39$} \\
\hline $\begin{array}{l}6.97 \\
7.15\end{array}$ & $\begin{array}{l}3.07 \times 10^{-18} \\
1.18 \times 10^{-18}\end{array}$ & $\tilde{r} / d_{\text {avg }}$ & $\mathcal{N}$ \\
\hline 7.33 & $4.54 \times 10^{-19}$ & 2.61 & $3.01 \times 10^{-8}$ \\
\hline 7.52 & $1.69 \times 10^{-19}$ & 2.77 & $1.26 \times 10^{-8}$ \\
\hline 7.7 & $5.99 \times 10^{-20}$ & 2.93 & $4.92 \times 10^{-9}$ \\
\hline 7.88 & $2.17 \times 10^{-20}$ & 3.09 & $2.00 \times 10^{-9}$ \\
\hline 8.06 & $8.33 \times 10^{-21}$ & 3.26 & $8.91 \times 10^{-10}$ \\
\hline 8.24 & $3.37 \times 10^{-21}$ & 3.42 & $4.13 \times 10^{-10}$ \\
\hline 8.42 & $1.40 \times 10^{-21}$ & 3.58 & $1.85 \times 10^{-10}$ \\
\hline & & 3.74 & $7.77 \times 10^{-11}$ \\
\hline \multicolumn{2}{|c|}{$d_{\text {lat }}=37$} & 3.9 & $3.11 \times 10^{-11}$ \\
\hline$\tilde{r} / d_{\text {avg }}$ & $\mathcal{N}$ & $\begin{array}{l}4.06 \\
4.22\end{array}$ & $\begin{array}{l}1.29 \times 10^{-11} \\
5.75 \times 10^{-12}\end{array}$ \\
\hline 2.62 & $2.79 \times 10^{-8}$ & 4.39 & $2.64 \times 10^{-12}$ \\
\hline 2.79 & $1.16 \times 10^{-8}$ & 4.55 & $1.18 \times 10^{-12}$ \\
\hline 2.96 & $4.39 \times 10^{-9}$ & 4.71 & $4.98 \times 10^{-13}$ \\
\hline 3.13 & $1.65 \times 10^{-9}$ & 4.87 & $2.03 \times 10^{-13}$ \\
\hline 3.3 & $6.91 \times 10^{-10}$ & 5.03 & $8.17 \times 10^{-14}$ \\
\hline 3.48 & $3.04 \times 10^{-10}$ & 5.19 & $3.46 \times 10^{-14}$ \\
\hline 3.65 & $1.29 \times 10^{-10}$ & 5.35 & $1.55 \times 10^{-14}$ \\
\hline 3.82 & $5.09 \times 10^{-11}$ & 5.52 & $7.04 \times 10^{-15}$ \\
\hline 3.99 & $1.91 \times 10^{-11}$ & 5.68 & $3.08 \times 10^{-15}$ \\
\hline 4.16 & $7.45 \times 10^{-12}$ & 5.84 & $1.31 \times 10^{-15}$ \\
\hline 4.33 & $3.14 \times 10^{-12}$ & 6. & $5.45 \times 10^{-16}$ \\
\hline 4.5 & $1.38 \times 10^{-12}$ & 6.16 & $2.28 \times 10^{-16}$ \\
\hline 4.67 & $5.96 \times 10^{-13}$ & 6.32 & $9.80 \times 10^{-17}$ \\
\hline 4.84 & $2.46 \times 10^{-13}$ & 6.48 & $4.32 \times 10^{-17}$ \\
\hline 5.01 & $9.52 \times 10^{-14}$ & 6.65 & $1.88 \times 10^{-17}$ \\
\hline 5.18 & $3.67 \times 10^{-14}$ & 6.81 & $7.94 \times 10^{-18}$ \\
\hline 5.36 & $1.52 \times 10^{-14}$ & 6.97 & $3.34 \times 10^{-18}$ \\
\hline 5.53 & $6.57 \times 10^{-15}$ & 7.13 & $1.42 \times 10^{-18}$ \\
\hline 5.7 & $2.76 \times 10^{-15}$ & 7.29 & $5.97 \times 10^{-19}$ \\
\hline 5.87 & $1.12 \times 10^{-15}$ & 7.45 & $2.48 \times 10^{-19}$ \\
\hline 6.04 & $4.50 \times 10^{-16}$ & 7.61 & $1.04 \times 10^{-19}$ \\
\hline 6.21 & $1.77 \times 10^{-16}$ & 7.78 & $4.40 \times 10^{-20}$ \\
\hline 6.38 & $6.96 \times 10^{-17}$ & 7.94 & $1.87 \times 10^{-20}$ \\
\hline 6.55 & $2.84 \times 10^{-17}$ & 8.1 & $8.05 \times 10^{-21}$ \\
\hline 6.72 & $1.19 \times 10^{-17}$ & 8.26 & $3.48 \times 10^{-21}$ \\
\hline 6.89 & $4.93 \times 10^{-18}$ & & \\
\hline 7.06 & $2.02 \times 10^{-18}$ & & \\
\hline 7.24 & $8.30 \times 10^{-19}$ & & \\
\hline 7.41 & $3.34 \times 10^{-19}$ & & \\
\hline 7.58 & $1.30 \times 10^{-19}$ & & \\
\hline 7.75 & $5.14 \times 10^{-20}$ & & \\
\hline 7.92 & $2.13 \times 10^{-20}$ & & \\
\hline 8.09 & $9.06 \times 10^{-21}$ & & \\
\hline
\end{tabular}

TABLE VIII. (Continued)

$1.26 \times 10^{-8}$

$4.92 \times 10^{-9}$

$2.00 \times 10^{-9}$

$8.91 \times 10^{-10}$

$4.13 \times 10^{-10}$

$1.85 \times 10^{-10}$

$3.11 \times 10^{-11}$

$5.75 \times 10^{-12}$

$2.64 \times 10^{-12}$

$1.18 \times 10^{-12}$

$4.98 \times 10^{-13}$

$8.17 \times 10^{-14}$

$3.46 \times 10^{-14}$

$1.55 \times 10^{-14}$

$7.04 \times 10^{-15}$

$3.08 \times 10^{-15}$

$1.31 \times 10^{-15}$

$5.45 \times 10^{-16}$

$2.28 \times 10^{-16}$

$4.32 \times 10^{-17}$

$1.88 \times 10^{-17}$

$7.94 \times 10^{-18}$

$3.34 \times 10^{-18}$

$1.42 \times 10^{-18}$

$5.97 \times 10^{-19}$

$1.87 \times 10^{-20}$

$8.05 \times 10^{-21}$

$3.48 \times 10^{-21}$
$1.29 \times 10^{-11}$

$1.04 \times 10^{-19}$

$4.40 \times 10^{-20}$ 
TABLE IX. Logarithmic negativity as a function of separation measured in units of the averaged region diameter with an $N$-type boundary appearing in the left panel in Fig. 3.

\begin{tabular}{|c|c|}
\hline \multicolumn{2}{|c|}{$d_{\text {lat }}=41$} \\
\hline$\tilde{r} / d_{\text {avg }}$ & $\mathcal{N}$ \\
\hline 2.6 & $3.24 \times 10^{-8}$ \\
\hline 2.8 & $1.04 \times 10^{-8}$ \\
\hline 3.01 & $3.20 \times 10^{-9}$ \\
\hline 3.21 & $1.13 \times 10^{-9}$ \\
\hline 3.41 & $4.28 \times 10^{-10}$ \\
\hline 3.62 & $1.51 \times 10^{-10}$ \\
\hline 3.82 & $4.76 \times 10^{-11}$ \\
\hline 4.02 & $1.55 \times 10^{-11}$ \\
\hline 4.23 & $5.74 \times 10^{-12}$ \\
\hline 4.43 & $2.16 \times 10^{-12}$ \\
\hline 4.63 & $7.38 \times 10^{-13}$ \\
\hline 4.84 & $2.30 \times 10^{-13}$ \\
\hline 5.04 & $7.40 \times 10^{-14}$ \\
\hline 5.25 & $2.71 \times 10^{-14}$ \\
\hline 5.45 & $1.02 \times 10^{-14}$ \\
\hline 5.65 & $3.52 \times 10^{-15}$ \\
\hline 5.86 & $1.14 \times 10^{-15}$ \\
\hline 6.06 & $3.67 \times 10^{-16}$ \\
\hline 6.26 & $1.27 \times 10^{-16}$ \\
\hline 6.47 & $4.65 \times 10^{-17}$ \\
\hline 6.67 & $1.63 \times 10^{-17}$ \\
\hline 6.88 & $5.37 \times 10^{-18}$ \\
\hline 7.08 & $1.78 \times 10^{-18}$ \\
\hline 7.28 & $6.05 \times 10^{-19}$ \\
\hline 7.49 & $2.09 \times 10^{-19}$ \\
\hline 7.69 & $7.12 \times 10^{-20}$ \\
\hline 7.89 & $2.33 \times 10^{-20}$ \\
\hline 8.1 & $7.48 \times 10^{-21}$ \\
\hline 8.3 & $2.54 \times 10^{-21}$ \\
\hline \multicolumn{2}{|c|}{$d_{\text {lat }}=43$} \\
\hline$\tilde{r} / d_{\text {avg }}$ & $\mathcal{N}$ \\
\hline 2.61 & $3.00 \times 10^{-8}$ \\
\hline 2.8 & $9.93 \times 10^{-9}$ \\
\hline 3. & $3.29 \times 10^{-9}$ \\
\hline 3.19 & $1.25 \times 10^{-9}$ \\
\hline 3.39 & $4.92 \times 10^{-10}$ \\
\hline 3.58 & $1.80 \times 10^{-10}$ \\
\hline 3.77 & $5.94 \times 10^{-11}$ \\
\hline 3.97 & $2.04 \times 10^{-11}$ \\
\hline 4.16 & $7.83 \times 10^{-12}$ \\
\hline 4.36 & $3.04 \times 10^{-12}$ \\
\hline 4.55 & $1.09 \times 10^{-12}$ \\
\hline 4.75 & $3.64 \times 10^{-13}$ \\
\hline 4.94 & $1.24 \times 10^{-13}$ \\
\hline 5.14 & $4.65 \times 10^{-14}$ \\
\hline 5.33 & $1.79 \times 10^{-14}$ \\
\hline 5.53 & $6.46 \times 10^{-15}$ \\
\hline
\end{tabular}

(Table continued)
TABLE IX. (Continued)

\begin{tabular}{|c|c|}
\hline \multicolumn{2}{|c|}{$d_{\text {lat }}=43$} \\
\hline$\tilde{r} / d_{\text {avg }}$ & $\mathcal{N}$ \\
\hline 5.72 & $2.21 \times 10^{-15}$ \\
\hline 5.92 & $7.55 \times 10^{-16}$ \\
\hline 6.11 & $2.75 \times 10^{-16}$ \\
\hline 6.31 & $1.06 \times 10^{-16}$ \\
\hline 6.5 & $3.96 \times 10^{-17}$ \\
\hline 6.7 & $1.36 \times 10^{-17}$ \\
\hline 6.89 & $4.68 \times 10^{-18}$ \\
\hline 7.09 & $1.68 \times 10^{-18}$ \\
\hline 7.28 & $6.17 \times 10^{-19}$ \\
\hline 7.48 & $2.30 \times 10^{-19}$ \\
\hline 7.67 & $8.35 \times 10^{-20}$ \\
\hline 7.87 & $2.90 \times 10^{-20}$ \\
\hline 8.06 & $9.85 \times 10^{-21}$ \\
\hline 8.26 & $3.46 \times 10^{-21}$ \\
\hline \multicolumn{2}{|c|}{$d_{\text {lat }}=47$} \\
\hline$\tilde{r} / d_{\text {avg }}$ & $\mathcal{N}$ \\
\hline 2.59 & $3.16 \times 10^{-8}$ \\
\hline 2.81 & $8.88 \times 10^{-9}$ \\
\hline 3.04 & $2.74 \times 10^{-9}$ \\
\hline 3.26 & $9.32 \times 10^{-10}$ \\
\hline 3.48 & $2.98 \times 10^{-10}$ \\
\hline 3.7 & $8.64 \times 10^{-11}$ \\
\hline 3.92 & $2.65 \times 10^{-11}$ \\
\hline 4.14 & $9.03 \times 10^{-12}$ \\
\hline 4.37 & $2.93 \times 10^{-12}$ \\
\hline 4.59 & $8.58 \times 10^{-13}$ \\
\hline 4.81 & $2.54 \times 10^{-13}$ \\
\hline 5.03 & $8.23 \times 10^{-14}$ \\
\hline 5.25 & $2.73 \times 10^{-14}$ \\
\hline 5.47 & $8.65 \times 10^{-15}$ \\
\hline 5.69 & $2.56 \times 10^{-15}$ \\
\hline 5.92 & $7.69 \times 10^{-16}$ \\
\hline 6.14 & $2.50 \times 10^{-16}$ \\
\hline 6.36 & $7.96 \times 10^{-17}$ \\
\hline 6.58 & $2.50 \times 10^{-17}$ \\
\hline 6.8 & $7.72 \times 10^{-18}$ \\
\hline 7.02 & $2.33 \times 10^{-18}$ \\
\hline 7.25 & $7.39 \times 10^{-19}$ \\
\hline 7.47 & $2.44 \times 10^{-19}$ \\
\hline 7.69 & $7.77 \times 10^{-20}$ \\
\hline 7.91 & $2.37 \times 10^{-20}$ \\
\hline 8.13 & $7.10 \times 10^{-21}$ \\
\hline \multicolumn{2}{|c|}{$d_{\text {lat }}=49$} \\
\hline$\tilde{r} / d_{\text {avg }}$ & $\mathcal{N}$ \\
\hline 2.59 & $3.05 \times 10^{-8}$ \\
\hline 2.76 & $1.15 \times 10^{-8}$ \\
\hline 2.93 & $4.57 \times 10^{-9}$ \\
\hline
\end{tabular}


TABLE IX. (Continued)

\begin{tabular}{|c|c|}
\hline \multicolumn{2}{|c|}{$d_{\text {lat }}=49$} \\
\hline$\tilde{r} / d_{\mathrm{avg}}$ & $\mathcal{N}$ \\
\hline 3.1 & $1.98 \times 10^{-9}$ \\
\hline 3.27 & $8.64 \times 10^{-10}$ \\
\hline 3.44 & $3.54 \times 10^{-10}$ \\
\hline 3.61 & $1.36 \times 10^{-10}$ \\
\hline 3.78 & $5.31 \times 10^{-11}$ \\
\hline 3.96 & $2.25 \times 10^{-11}$ \\
\hline 4.13 & $9.87 \times 10^{-12}$ \\
\hline 4.3 & $4.16 \times 10^{-12}$ \\
\hline 4.47 & $1.65 \times 10^{-12}$ \\
\hline 4.64 & $6.37 \times 10^{-13}$ \\
\hline 4.81 & $2.58 \times 10^{-13}$ \\
\hline 4.98 & $1.11 \times 10^{-13}$ \\
\hline 5.15 & $4.75 \times 10^{-14}$ \\
\hline 5.32 & $1.95 \times 10^{-14}$ \\
\hline 5.49 & $7.73 \times 10^{-15}$ \\
\hline 5.66 & $2.99 \times 10^{-15}$ \\
\hline 5.83 & $1.21 \times 10^{-15}$ \\
\hline 6. & $5.17 \times 10^{-16}$ \\
\hline 6.17 & $2.22 \times 10^{-16}$ \\
\hline 6.34 & $9.13 \times 10^{-17}$ \\
\hline 6.51 & $3.66 \times 10^{-17}$ \\
\hline 6.68 & $1.47 \times 10^{-17}$ \\
\hline 6.85 & $5.99 \times 10^{-18}$ \\
\hline 7.02 & $2.49 \times 10^{-18}$ \\
\hline 7.19 & $1.05 \times 10^{-18}$ \\
\hline 7.36 & $4.35 \times 10^{-19}$ \\
\hline 7.53 & $1.79 \times 10^{-19}$ \\
\hline 7.7 & $7.23 \times 10^{-20}$ \\
\hline 7.87 & $2.86 \times 10^{-20}$ \\
\hline 8.04 & $1.14 \times 10^{-20}$ \\
\hline 8.21 & $4.69 \times 10^{-21}$ \\
\hline
\end{tabular}

TABLE X. Logarithmic negativity as a function of separation measured in units of the averaged region diameter with an $N$-type boundary appearing in the left panel in Fig. 3.

\begin{tabular}{lc}
\hline \hline & $d_{\text {lat }}=57$ \\
\hline$\tilde{r} / d_{\text {avg }}$ & $\mathcal{N}$ \\
\hline 2.58 & $3.22 \times 10^{-8}$ \\
2.76 & $1.17 \times 10^{-8}$ \\
2.94 & $4.71 \times 10^{-9}$ \\
3.12 & $1.93 \times 10^{-9}$ \\
3.3 & $7.43 \times 10^{-10}$ \\
3.48 & $2.68 \times 10^{-10}$ \\
3.66 & $1.01 \times 10^{-10}$ \\
3.85 & $4.09 \times 10^{-11}$ \\
4.03 & $1.66 \times 10^{-11}$ \\
\hline & (Table continued)
\end{tabular}

TABLE X. (Continued)

\begin{tabular}{|c|c|}
\hline \multicolumn{2}{|c|}{$d_{\text {lat }}=57$} \\
\hline$\tilde{r} / d_{\text {avg }}$ & $\mathcal{N}$ \\
\hline 4.21 & $6.31 \times 10^{-12}$ \\
\hline 4.39 & $2.30 \times 10^{-12}$ \\
\hline 4.57 & $8.73 \times 10^{-13}$ \\
\hline 4.75 & $3.52 \times 10^{-13}$ \\
\hline 4.93 & $1.42 \times 10^{-13}$ \\
\hline 5.11 & $5.45 \times 10^{-14}$ \\
\hline 5.3 & $2.02 \times 10^{-14}$ \\
\hline 5.48 & $7.49 \times 10^{-15}$ \\
\hline 5.66 & $2.96 \times 10^{-15}$ \\
\hline 5.84 & $1.21 \times 10^{-15}$ \\
\hline 6.02 & $4.81 \times 10^{-16}$ \\
\hline 6.2 & $1.84 \times 10^{-16}$ \\
\hline 6.38 & $6.89 \times 10^{-17}$ \\
\hline 6.57 & $2.61 \times 10^{-17}$ \\
\hline 6.75 & $1.02 \times 10^{-17}$ \\
\hline 6.93 & $4.01 \times 10^{-18}$ \\
\hline 7.11 & $1.55 \times 10^{-18}$ \\
\hline 7.29 & $5.96 \times 10^{-19}$ \\
\hline 7.47 & $2.25 \times 10^{-19}$ \\
\hline 7.65 & $8.42 \times 10^{-20}$ \\
\hline 7.84 & $3.25 \times 10^{-20}$ \\
\hline 8.02 & $1.29 \times 10^{-20}$ \\
\hline 8.2 & $5.11 \times 10^{-21}$ \\
\hline \multicolumn{2}{|c|}{$d_{\text {lat }}=67$} \\
\hline$\tilde{r} / d_{\text {avg }}$ & $\mathcal{N}$ \\
\hline 2.56 & $3.28 \times 10^{-8}$ \\
\hline 2.75 & $1.26 \times 10^{-8}$ \\
\hline 2.93 & $5.04 \times 10^{-9}$ \\
\hline 3.12 & $1.93 \times 10^{-9}$ \\
\hline 3.3 & $6.89 \times 10^{-10}$ \\
\hline 3.49 & $2.54 \times 10^{-10}$ \\
\hline 3.67 & $1.01 \times 10^{-10}$ \\
\hline 3.85 & $4.00 \times 10^{-11}$ \\
\hline 4.04 & $1.49 \times 10^{-11}$ \\
\hline 4.22 & $5.37 \times 10^{-12}$ \\
\hline 4.41 & $2.05 \times 10^{-12}$ \\
\hline 4.59 & $8.22 \times 10^{-13}$ \\
\hline 4.78 & $3.21 \times 10^{-13}$ \\
\hline 4.96 & $1.18 \times 10^{-13}$ \\
\hline 5.14 & $4.27 \times 10^{-14}$ \\
\hline 5.33 & $1.63 \times 10^{-14}$ \\
\hline 5.51 & $6.49 \times 10^{-15}$ \\
\hline 5.7 & $2.55 \times 10^{-15}$ \\
\hline 5.88 & $9.57 \times 10^{-16}$ \\
\hline 6.07 & $3.51 \times 10^{-16}$ \\
\hline 6.25 & $1.32 \times 10^{-16}$ \\
\hline 6.43 & $5.12 \times 10^{-17}$ \\
\hline 6.62 & $1.99 \times 10^{-17}$ \\
\hline 6.8 & $7.60 \times 10^{-18}$ \\
\hline
\end{tabular}


TABLE X. (Continued)

\begin{tabular}{|c|c|}
\hline \multicolumn{2}{|c|}{$d_{\text {lat }}=67$} \\
\hline$\tilde{r} / d_{\mathrm{avg}}$ & $\mathcal{N}$ \\
\hline 6.99 & $2.87 \times 10^{-18}$ \\
\hline 7.17 & $1.08 \times 10^{-18}$ \\
\hline 7.36 & $4.13 \times 10^{-19}$ \\
\hline 7.54 & $1.60 \times 10^{-19}$ \\
\hline 7.72 & $6.14 \times 10^{-20}$ \\
\hline 7.91 & $2.33 \times 10^{-20}$ \\
\hline 8.09 & $8.66 \times 10^{-21}$ \\
\hline \multicolumn{2}{|c|}{$d_{\text {lat }}=79$} \\
\hline$\tilde{r} / d_{\mathrm{avg}}$ & $\mathcal{N}$ \\
\hline 2.55 & $3.54 \times 10^{-8}$ \\
\hline 2.76 & $1.25 \times 10^{-8}$ \\
\hline 2.96 & $4.26 \times 10^{-9}$ \\
\hline 3.17 & $1.35 \times 10^{-9}$ \\
\hline 3.38 & $4.46 \times 10^{-10}$ \\
\hline 3.59 & $1.58 \times 10^{-10}$ \\
\hline 3.79 & $5.38 \times 10^{-11}$ \\
\hline 4. & $1.72 \times 10^{-11}$ \\
\hline 4.21 & $5.75 \times 10^{-12}$ \\
\hline 4.42 & $2.04 \times 10^{-12}$ \\
\hline 4.62 & $7.00 \times 10^{-13}$ \\
\hline 4.83 & $2.25 \times 10^{-13}$ \\
\hline 5.04 & $7.37 \times 10^{-14}$ \\
\hline 5.24 & $2.59 \times 10^{-14}$ \\
\hline 5.45 & $8.97 \times 10^{-15}$ \\
\hline 5.66 & $2.96 \times 10^{-15}$ \\
\hline 5.87 & $9.68 \times 10^{-16}$ \\
\hline 6.07 & $3.31 \times 10^{-16}$ \\
\hline 6.28 & $1.15 \times 10^{-16}$ \\
\hline 6.49 & $3.94 \times 10^{-17}$ \\
\hline 6.69 & $1.31 \times 10^{-17}$ \\
\hline 6.9 & $4.32 \times 10^{-18}$ \\
\hline 7.11 & $1.48 \times 10^{-18}$ \\
\hline 7.32 & $5.05 \times 10^{-19}$ \\
\hline 7.52 & $1.71 \times 10^{-19}$ \\
\hline 7.73 & $5.63 \times 10^{-20}$ \\
\hline 7.94 & $1.87 \times 10^{-20}$ \\
\hline 8.14 & $6.35 \times 10^{-21}$ \\
\hline
\end{tabular}

TABLE XI. Negativity decay constants $\beta_{2 D}$ extracted at finite region pixelation appearing in the middle two panels in Fig. 3.

\begin{tabular}{lr}
\hline \hline $1 / d_{\text {lat }}$ & \\
\hline$\frac{1}{21}$ & $\beta_{2 D}$ \\
$\frac{1}{23}$ & $5.955(80)$ \\
$\frac{1}{25}$ & $5.908(61)$ \\
& $5.823(40)$ \\
\hline
\end{tabular}

TABLE XI. (Continued)

\begin{tabular}{|c|c|}
\hline \multicolumn{2}{|c|}{$N$ boundary } \\
\hline $1 / d_{\text {lat }}$ & $\beta_{2 D}$ \\
\hline $\begin{array}{l}\frac{1}{27} \\
\frac{1}{29} \\
\frac{1}{31} \\
\frac{1}{33} \\
\frac{1}{35} \\
\frac{1}{37} \\
\frac{1}{39} \\
\frac{1}{41} \\
\frac{1}{43} \\
\frac{1}{47} \\
\frac{1}{49} \\
\frac{1}{57} \\
\frac{1}{67} \\
\frac{1}{79}\end{array}$ & $\begin{array}{l}5.698(26) \\
5.633(17) \\
5.628(23) \\
5.598(16) \\
5.574(13) \\
5.530(10) \\
5.514(11) \\
5.494(15) \\
5.497(12) \\
5.459(10) \\
5.460(8) \\
5.419(7) \\
5.392(6) \\
5.367(6)\end{array}$ \\
\hline \multicolumn{2}{|c|}{$S$ boundary } \\
\hline $1 / d_{\text {lat }}$ & $\beta_{2 D}$ \\
\hline $\begin{array}{l}\frac{1}{20} \\
\frac{1}{22} \\
\frac{1}{24} \\
\frac{1}{26} \\
\frac{1}{28} \\
\frac{1}{30} \\
\frac{1}{32} \\
\frac{1}{34} \\
\frac{1}{36} \\
\frac{1}{38} \\
\frac{1}{40} \\
\frac{1}{42} \\
\frac{1}{44} \\
\frac{1}{48} \\
\frac{1}{56} \\
\frac{1}{58} \\
\frac{1}{68} \\
\frac{1}{80}\end{array}$ & $\begin{array}{c}5.680(56) \\
5.556(34) \\
5.540(17) \\
5.475(35) \\
5.457(13) \\
5.408(21) \\
5.380(8) \\
5.392(16) \\
5.380(14) \\
5.394(6) \\
5.344(10) \\
5.329(11) \\
5.340(11) \\
5.334(11) \\
5.304(10) \\
5.315(7) \\
5.300(7) \\
5.299(7)\end{array}$ \\
\hline \multicolumn{2}{|c|}{$N$ boundary } \\
\hline $1 / d_{\mathrm{avg}}$ & $\beta_{2 D}$ \\
\hline 0.052 & $5.428(63)$ \\
\hline 0.047 & $5.410(70)$ \\
\hline 0.044 & $5.308(32)$ \\
\hline 0.04 & $5.310(25)$ \\
\hline 0.037 & $5.278(17)$ \\
\hline 0.034 & $5.307(23)$ \\
\hline 0.032 & $5.271(17)$ \\
\hline 0.03 & $5.270(13)$ \\
\hline 0.028 & $5.249(11)$ \\
\hline
\end{tabular}


TABLE XI. (Continued)

\begin{tabular}{|c|c|}
\hline \multicolumn{2}{|c|}{$N$ boundary } \\
\hline $1 / d_{\mathrm{avg}}$ & $\beta_{2 D}$ \\
\hline 0.027 & $5.251(12)$ \\
\hline 0.025 & $5.253(14)$ \\
\hline 0.024 & $5.244(12)$ \\
\hline 0.022 & $5.240(10)$ \\
\hline 0.021 & $5.237(9)$ \\
\hline 0.018 & $5.241(8)$ \\
\hline 0.015 & $5.240(7)$ \\
\hline 0.013 & $5.247(6)$ \\
\hline \multicolumn{2}{|c|}{$S$ boundary } \\
\hline $1 / d_{\mathrm{avg}}$ & $\beta_{2 D}$ \\
\hline 0.052 & $5.466(54)$ \\
\hline 0.047 & $5.350(31)$ \\
\hline 0.043 & $5.323(18)$ \\
\hline 0.039 & $5.331(31)$ \\
\hline 0.037 & $5.295(13)$ \\
\hline 0.034 & $5.287(19)$ \\
\hline 0.032 & $5.264(7)$ \\
\hline 0.03 & $5.274(17)$ \\
\hline 0.028 & $5.262(15)$ \\
\hline 0.027 & $5.260(6)$ \\
\hline 0.025 & $5.251(10)$ \\
\hline 0.024 & $5.246(11)$ \\
\hline 0.023 & $5.254(12)$ \\
\hline 0.021 & $5.238(10)$ \\
\hline 0.018 & $5.238(10)$ \\
\hline 0.017 & $5.245(8)$ \\
\hline 0.015 & $5.243(7)$ \\
\hline 0.013 & $5.245(8)$ \\
\hline
\end{tabular}

TABLE XII. Entanglement bubble radii for regions of the onedimensional massless scalar field appearing in the right panel in Fig. 3.

\begin{tabular}{lc}
\hline \hline$d_{\text {lat }}$ & $\tilde{r}_{\mathcal{X}} / d_{\text {lat }}$ \\
\hline 1 & 1.000 \\
2 & 1.000 \\
3 & 3.000 \\
4 & 3.250 \\
5 & 5.200 \\
6 & 5.333 \\
7 & 7.429 \\
8 & 7.625 \\
9 & 9.667 \\
10 & 9.800 \\
11 & 11.91 \\
12 & 12.00 \\
13 & 14.15 \\
14 & 14.21 \\
15 & 16.33 \\
\hline
\end{tabular}

TABLE XII. (Continued)

\begin{tabular}{|c|c|}
\hline$d_{\text {lat }}$ & $\tilde{r}_{\mathcal{X}} / d_{\text {lat }}$ \\
\hline 16 & 16.50 \\
\hline 17 & 18.59 \\
\hline 18 & 18.72 \\
\hline 19 & 20.79 \\
\hline 20 & 20.95 \\
\hline 21 & 23.05 \\
\hline 22 & 23.18 \\
\hline 23 & 25.26 \\
\hline 24 & 25.42 \\
\hline 25 & 27.52 \\
\hline 26 & 27.65 \\
\hline 27 & 29.74 \\
\hline 28 & 29.86 \\
\hline 29 & 32.00 \\
\hline 30 & 32.10 \\
\hline 31 & 34.23 \\
\hline 32 & 34.34 \\
\hline 33 & 36.45 \\
\hline 34 & 36.59 \\
\hline 35 & 38.69 \\
\hline 36 & 38.81 \\
\hline 37 & 40.92 \\
\hline 38 & 41.05 \\
\hline 39 & 43.15 \\
\hline 40 & 43.30 \\
\hline 41 & 45.39 \\
\hline 42 & 45.52 \\
\hline 43 & 47.63 \\
\hline 44 & 47.77 \\
\hline 45 & 49.87 \\
\hline 46 & 50.00 \\
\hline 47 & 52.11 \\
\hline 48 & 52.25 \\
\hline 49 & 54.35 \\
\hline 50 & 54.48 \\
\hline 51 & 56.59 \\
\hline 52 & 56.71 \\
\hline 53 & 58.83 \\
\hline 54 & 58.94 \\
\hline 55 & 61.07 \\
\hline 56 & 61.20 \\
\hline 57 & 63.30 \\
\hline 58 & 63.43 \\
\hline 59 & 65.54 \\
\hline 60 & 65.67 \\
\hline 61 & 67.77 \\
\hline 62 & 67.90 \\
\hline 63 & 70.02 \\
\hline 64 & 70.14 \\
\hline 65 & 72.25 \\
\hline 66 & 72.38 \\
\hline 67 & 74.49 \\
\hline 68 & 74.62 \\
\hline 69 & 76.72 \\
\hline 70 & 76.86 \\
\hline 71 & 78.97 \\
\hline
\end{tabular}


TABLE XII. (Continued)

\begin{tabular}{|c|c|c|c|}
\hline$d_{\text {lat }}$ & $\tilde{r}_{\mathcal{X}} / d_{\text {lat }}$ & & \\
\hline 72 & 79.10 & \multicolumn{2}{|c|}{$N$ boundary } \\
\hline 74 & $\begin{array}{l}01.21 \\
81.32\end{array}$ & $\overline{d_{\text {lat }}}$ & $\tilde{r}_{\mathcal{X}} / d_{\text {lat }}$ \\
\hline 75 & 83.44 & 3 & 1.33 \\
\hline 76 & 83.57 & 5 & 2.00 \\
\hline 77 & 85.68 & 7 & 3.00 \\
\hline 78 & 85.81 & 9 & 4.11 \\
\hline 79 & 87.91 & 11 & 5.36 \\
\hline 80 & 88.05 & 13 & 6.46 \\
\hline 81 & 90.16 & 15 & 7.47 \\
\hline 82 & 90.28 & 17 & 8.65 \\
\hline 83 & 92.40 & 21 & 11.4 \\
\hline 84 & 92.52 & 25 & 13.6 \\
\hline 85 & 94.64 & $2 J$ & \\
\hline 86 & 94.76 & \multirow{2}{*}{\multicolumn{2}{|c|}{$S$ boundary (even) }} \\
\hline 87 & 96.87 & & \\
\hline 88 & 97.00 & $d_{\text {lat }}$ & $\tilde{r}_{\mathcal{X}} / d_{\text {lat }}$ \\
\hline 89 & 99.10 & $\overline{4}$ & 1.50 \\
\hline 90 & 99.23 & 6 & 3.00 \\
\hline 91 & 101.3 & 8 & 4.00 \\
\hline 92 & 101.5 & 10 & 5.20 \\
\hline 93 & 103.6 & 12 & 6.42 \\
\hline 94 & 103.7 & 14 & 7.64 \\
\hline 95 & 105.8 & 16 & 9.00 \\
\hline 96 & 105.9 & 18 & 10.2 \\
\hline $\begin{array}{l}97 \\
98\end{array}$ & 108.1 & & \\
\hline $\begin{array}{l}98 \\
99\end{array}$ & $\begin{array}{l}108.2 \\
110.3\end{array}$ & \multicolumn{2}{|c|}{$S$ boundary (odd) } \\
\hline 100 & 110.4 & $d_{\text {lat }}$ & $\tilde{r}_{\mathcal{X}} / d_{\text {lat }}$ \\
\hline 101 & 112.5 & & 167 \\
\hline 102 & 112.7 & $\begin{array}{l}3 \\
5\end{array}$ & $\begin{array}{l}1.67 \\
260\end{array}$ \\
\hline 103 & 114.8 & 5 & 2.60 \\
\hline 104 & 114.9 & 7 & 3.71 \\
\hline 105 & 117.0 & 9 & 5.11 \\
\hline 106 & 117.1 & 11 & 6.27 \\
\hline 107 & 119.3 & 13 & 7.62 \\
\hline 108 & 119.4 & 15 & 8.80 \\
\hline 109 & 121.5 & 17 & 10.0 \\
\hline 110 & 121.6 & & \\
\hline 111 & 123.7 & & \\
\hline 112 & 123.8 & & \\
\hline 113 & 126.0 & & \\
\hline 114 & 126.1 & & \\
\hline 115 & 128.2 & & \\
\hline
\end{tabular}

TABLE XIII. Entanglement bubble radii for regions of the twodimensional massless scalar field appearing in the right panel in Fig. 3.
[1] H. Reeh and S. Schlieder, Il Nuovo Cimento (1955-1965) 22, 1051 (1961).

[2] S. J. Summers and R. Werner, Phys. Lett. A 110, 257 (1985).
[3] S. J. Summers and R. Werner, J. Math. Phys. (N.Y.) 28, 2440 (1987).

[4] S. J. Summers and R. Werner, J. Math. Phys. (N.Y.) 28, 2448 (1987). 
[5] E. Witten, Rev. Mod. Phys. 90, 045003 (2018).

[6] A. Valentini, Phys. Lett. A 153, 321 (1991).

[7] B. Reznik, Found. Phys. 33, 167 (2003).

[8] B. Reznik, A. Retzker, and J. Silman, Phys. Rev. A 71, 042104 (2005).

[9] G. Salton, R. B. Mann, and N. C. Menicucci, New J. Phys. 17, 035001 (2015).

[10] A. Pozas-Kerstjens and E. Martin-Martinez, Phys. Rev. D 92, 064042 (2015).

[11] S. Ryu and T. Takayanagi, Phys. Rev. Lett. 96, 181602 (2006).

[12] S. Ryu and T. Takayanagi, J. High Energy Phys. 08 (2006) 045.

[13] G. L. Ver Steeg and N. C. Menicucci, Phys. Rev. D 79, 044027 (2009).

[14] E. Martín-Martínez and N. C. Menicucci, Classical Quantum Gravity 29, 224003 (2012).

[15] E. Martin-Martinez, A. R. H. Smith, and D. R. Terno, Phys. Rev. D 93, 044001 (2016).

[16] B. Swingle, Annu. Rev. Condens. Matter Phys. 9, 345 (2018).

[17] J. Berges, S. Floerchinger, and R. Venugopalan, Phys. Lett. B 778, 442 (2018).

[18] J. Berges, S. Floerchinger, and R. Venugopalan, J. High Energy Phys. 04 (2018) 145.

[19] J. Berges, S. Floerchinger, and R. Venugopalan, Nucl. Phys. A982, 819 (2019).

[20] C. M. Ho and S. D. H. Hsu, Mod. Phys. Lett. A 31, 1650110 (2016).

[21] A. Kovner and M. Lublinsky, Phys. Rev. D 92, 034016 (2015).

[22] A. Kovner, M. Lublinsky, and M. Serino, Phys. Lett. B 792, 4 (2019).

[23] N. Armesto, F. Dominguez, A. Kovner, M. Lublinsky, and V. Skokov, J. High Energy Phys. 05 (2019) 025.

[24] D. E. Kharzeev and E. M. Levin, Phys. Rev. D 95, 114008 (2017).

[25] Z. Tu, D. E. Kharzeev, and T. Ullrich, Phys. Rev. Lett. 124, 062001 (2020).

[26] O. K. Baker and D. E. Kharzeev, Phys. Rev. D 98, 054007 (2018).

[27] S. R. Beane, D. B. Kaplan, N. Klco, and M. J. Savage, Phys. Rev. Lett. 122, 102001 (2019).

[28] S. R. Beane and P. Ehlers, Mod. Phys. Lett. A 35, 2050048 (2020).

[29] O. C. Gorton, Master's thesis, San Diego State University, 2018.

[30] O. Gorton and C. W. Johnson, Entanglement entropy and proton-neutron interactions (2019), ESNT workshop on proton-neutron pairing, http://esnt.cea.fr/Phocea/Page/ index.php?id=84.

[31] C. Robin, M. J. Savage, and N. Pillet, arXiv:2007.09157.

[32] S. P. Jordan, K. S. M. Lee, and J. Preskill, Quantum Inf. Comput. 14, 1014 (2014).

[33] S. P. Jordan, K. S. M. Lee, and J. Preskill, Science 336, 1130 (2012).

[34] K. Yeter-Aydeniz and G. Siopsis, Phys. Rev. D 97, 036004 (2018).

[35] N. Klco and M. J. Savage, Phys. Rev. A 99, 052335 (2019).
[36] K. Yeter-Aydeniz, E. F. Dumitrescu, A. J. McCaskey, R. S. Bennink, R. C. Pooser, and G. Siopsis, Phys. Rev. A 99, 032306 (2019).

[37] S. P. Jordan, H. Krovi, K. S. M. Lee, and J. Preskill, Quantum 2, 44 (2018).

[38] M. Srednicki, Phys. Rev. Lett. 71, 666 (1993).

[39] K. Audenaert, J. Eisert, M. Plenio, and R. Werner, Phys. Rev. A 66, 042327 (2002).

[40] A. Botero and B. Reznik, Phys. Rev. A 70, 052329 (2004).

[41] J. Kofler, V. Vedral, M. S. Kim, and Č. Brukner, Phys. Rev. A 73, 052107 (2006).

[42] S. Marcovitch, A. Retzker, M. Plenio, and B. Reznik, Phys. Rev. A 80, 012325 (2009).

[43] R. Lohmayer, H. Neuberger, A. Schwimmer, and S. Theisen, Phys. Lett. B 685, 222 (2010).

[44] P. Calabrese, J. Cardy, and E. Tonni, J. Stat. Mech. (2009) P11001.

[45] H. Casini and M. Huerta, Phys. Lett. B 694, 167 (2010).

[46] P. Calabrese, J. Cardy, and E. Tonni, Phys. Rev. Lett. 109, 130502 (2012).

[47] P. Calabrese, J. Cardy, and E. Tonni, J. Stat. Mech. (2013) P02008.

[48] M. R. Mohammadi Mozaffar and A. Mollabashi, J. High Energy Phys. 07 (2017) 120.

[49] A. Coser, C. De Nobili, and E. Tonni, J. Phys. A 50, 314001 (2017).

[50] G. Di Giulio and E. Tonni, J. Stat. Mech. (2020) 033102.

[51] H. Politzer, Phys. Rev. Lett. 30, 1346 (1973).

[52] D. J. Gross and F. Wilczek, Phys. Rev. Lett. 30, 1343 (1973).

[53] K. G. Wilson, Phys. Rev. D 10, 2445 (1974).

[54] M. Creutz, L. Jacobs, and C. Rebbi, Phys. Rev. Lett. 42, 1390 (1979).

[55] R. Balian, J. Drouffe, and C. Itzykson, Phys. Rev. D 10, 3376 (1974).

[56] S. R. Beane, E. Chang, S. D. Cohen, W. Detmold, H. W. Lin, T. C. Luu, K. Orginos, A. Parreño, M. J. Savage, and A. Walker-Loud (NPLQCD Collaboration), Phys. Rev. D 87, 034506 (2013).

[57] S. Aoki, T. Doi, T. Hatsuda, Y. Ikeda, T. Inoue, N. Ishii, K. Murano, H. Nemura, and K. Sasaki (HALQCD Collaboration), Prog. Theor. Exp. Phys. 2012, 01 A105 (2012).

[58] T. Yamazaki, K.-I. Ishikawa, Y. Kuramashi, and A. Ukawa, Phys. Rev. D 92, 014501 (2015).

[59] M. L. Wagman, F. Winter, E. Chang, Z. Davoudi, W. Detmold, K. Orginos, M. J. Savage, and P. E. Shanahan (NPLQCD Collaboration), Phys. Rev. D 96, 114510 (2017).

[60] S. Weinberg, Phys. Lett. B 251, 288 (1990).

[61] S. Weinberg, Nucl. Phys. B363, 3 (1991).

[62] U. van Kolck, Phys. Rev. C 49, 2932 (1994).

[63] D. B. Kaplan, M. J. Savage, and M. B. Wise, Phys. Lett. B 424, 390 (1998).

[64] E. Epelbaum, H. Krebs, and P. Reinert, Front. Phys. 8, 98 (2020).

[65] M. Horodecki, P. Horodecki, and R. Horodecki, Phys. Lett. A 223, 1 (1996).

[66] A. Peres, Phys. Rev. Lett. 77, 1413 (1996).

[67] G. Vidal and R. Werner, Phys. Rev. A 65, 032314 (2002).

[68] R. Simon, Phys. Rev. Lett. 84, 2726 (2000). 
[69] M. Horodecki, P. Horodecki, and R. Horodecki, Phys. Rev. Lett. 80, 5239 (1998).

[70] W. Cong, E. Tjoa, and R. B. Mann, J. High Energy Phys. 06 (2019) 021; 07 (2019) 051(E).

[71] L. J. Henderson, A. Belenchia, E. Castro-Ruiz, C. Budroni, M. Zych, v. C. Brukner, and R. B. Mann, Phys. Rev. Lett. 125, 131602 (2020).

[72] M. Horodecki, P. Horodecki, and R. Horodecki, Phys. Rev. Lett. 84, 4260 (2000).

[73] N. Klco and M. J. Savage, Phys. Rev. A 102, 012619 (2020).

[74] T. Yu and J. H. Eberly, Phys. Rev. Lett. 93, 140404 (2004).

[75] P. J. Dodd and J. J. Halliwell, Phys. Rev. A 69, 052105 (2004).

[76] M. P. Almeida, F. de Melo, M. Hor-Meyll, A. Salles, S. P. Walborn, P. H. S. Ribeiro, and L. Davidovich, Science 316, 579 (2007).

[77] T. Yu and J. H. Eberly, Science 323, 598 (2009).

[78] Z. Davoudi and M. J. Savage, Phys. Rev. D 86, 054505 (2012).

[79] K. Symanzik, Nucl. Phys. B226, 187 (1983).

[80] T. Luu and M. J. Savage, Phys. Rev. D 83, 114508 (2011).

[81] G. Adesso, A. Serafini, and F. Illuminati, Phys. Rev. A 70, 022318 (2004).

[82] M. M. Wolf, G. Giedke, and J. I. Cirac, Phys. Rev. Lett. 96, 080502 (2006).

[83] H. Muller, S. Koonin, R. Seki, and U. van Kolck, Phys. Rev. C 61, 044320 (2000).

[84] T. A. Lahde and U.-G. Meissner, Nuclear Lattice Effective Field Theory: An Introduction, Vol. 957 (Springer, New York, 2019).

[85] D. Lee, Front. Phys. 8, 174 (2020).

[86] D. Lee, Prog. Part. Nucl. Phys. 63, 117 (2009).

[87] G. 't Hooft, Nucl. Phys. B72, 461 (1974).

[88] E. Witten, Ann. Phys. (N.Y.) 128, 363 (1980).

[89] R. F. Dashen, E. E. Jenkins, and A. V. Manohar, Phys. Rev. D 49, 4713 (1994); 51, 2489(E) (1995).
[90] R. F. Dashen, E. E. Jenkins, and A. V. Manohar, Phys. Rev. D 51, 3697 (1995).

[91] D. B. Kaplan and M. J. Savage, Phys. Lett. B 365, 244 (1996).

[92] D. B. Kaplan and A. V. Manohar, Phys. Rev. C 56, 76 (1997).

[93] V. Coffman, J. Kundu, and W. K. Wootters, Phys. Rev. A 61, 052306 (2000).

[94] W. Dur, G. Vidal, and J. Cirac, Phys. Rev. A 62, 062314 (2000).

[95] R. Lohmayer, A. Osterloh, J. Siewert, and A. Uhlmann, Phys. Rev. Lett. 97, 260502 (2006).

[96] M. Rangamani and M. Rota, J. Phys. A 48, 385301 (2015).

[97] M. Lüscher, Commun. Math. Phys. 105, 153 (1986).

[98] M. Lüscher, Nucl. Phys. B354, 531 (1991).

[99] M. Lüscher and U. Wolff, Nucl. Phys. B339, 222 (1990).

[100] E. E. Jenkins and A. V. Manohar, Phys. Lett. B 255, 558 (1991).

[101] S. Beane, P. F. Bedaque, M. Savage, and U. van Kolck, Nucl. Phys. A700, 377 (2002).

[102] M. C. Birse, Phys. Rev. C 74, 014003 (2006).

[103] D. B. Kaplan, Phys. Rev. C 102, 034004 (2020).

[104] R. D. Young, D. B. Leinweber, and A. W. Thomas, Nucl. Phys. B, Proc. Suppl. 141, 233 (2005).

[105] S. R. White, Phys. Rev. Lett. 69, 2863 (1992).

[106] G. Vidal, Phys. Rev. Lett. 91, 147902 (2003).

[107] U. Schollwöck, Rev. Mod. Phys. 77, 259 (2005).

[108] F. Verstraete, V. Murg, and J. I. Cirac, Adv. Phys. 57, 143 (2008).

[109] Wolfram Research, Inc., Mathematica, Version 11.1, Champaign, IL, 2020.

[110] Advanpix LLC., Multiprecision computing toolbox for MATLAB.

[111] MATLAB, version 9.8.0.1396136 (R2020a) update 3 (The MathWorks Inc., Natick, Massachusetts, 2020). 